\title{
On the partial differential equations of electrostatic MEMS devices II: Dynamic case
}

\author{
Nassif GHOUSSOUB* and Yujin GUO ${ }^{\dagger}$ \\ Department of Mathematics \\ University of British Columbia \\ Vancouver V6T 1Z2, B.C., Canada \\ e-mail: nassif@math.ubc.ca and yjguo@math.umn.edu
}

\begin{abstract}
This paper is a continuation of [9], where we analyzed steadystates of the nonlinear parabolic problem $u_{t}=\Delta u-\frac{\lambda f(x)}{(1+u)^{2}}$ on a bounded domain $\Omega$ of $\mathbb{R}^{N}$ with Dirichlet boundary conditions. This equation models a simple electrostatic Micro-Electromechanical System (MEMS) device consisting of a thin dielectric elastic membrane with boundary supported at 0 above a rigid ground plate located at -1 . Here $u$ is modeled to describe dynamic deflection of the elastic membrane. When a voltage-represented here by $\lambda$ - is applied, the membrane deflects towards the ground plate and a snap-through (touchdown) may occur when it exceeds a certain critical value $\lambda^{*}$ (pull-in voltage), creating a so-called "pull-in instability" which greatly affects the design of many devices. In an effort to achieve better MEMS designs, the material properties of the membrane can be technologically fabricated with a spatially varying dielectric permittivity profile $f(x)$. We show that when $\lambda \leq \lambda^{*}$ the membrane globally converges to its unique maximal steady-state. On the other hand, if $\lambda>\lambda^{*}$ the membrane must touchdown at finite time $T$, and that touchdown cannot occur at a location where the permittivity profile vanishes. We establish upper and lower bounds on first touchdown times, and we analyze their dependence on $f, \lambda$ and $\Omega$ by applying various analytical and numerical techniques. A refined description of MEMS touchdown profiles is given in a companion paper [10].
\end{abstract}

2000 Mathematics Subject Classification: 35K55, 35K65.

Key words: MEMS; steady-state; global convergence; touchdown points; touchdown time.

\footnotetext{
* Partially supported by the Natural Science and Engineering Research Council of Canada. ${ }^{\dagger}$ Partially supported by a U.B.C. Graduate Fellowship.
} 


\section{Introduction}

Micro-Electromechanical Systems (MEMS) are often used to combine electronics with micro-size mechanical devices in the design of various types of microscopic machinery. MEMS devices have therefore become key components of many commercial systems, including accelerometers for airbag deployment in automobiles, ink jet printer heads, optical switches, chemical sensors, and so on. The simplicity and importance of this technique have led many applied mathematicians and engineers to study mathematical models of electrostatic-elastic interactions. An overview of the physical phenomena of the mathematical models associated with the rapidly developing field of MEMS technology is given in [16].

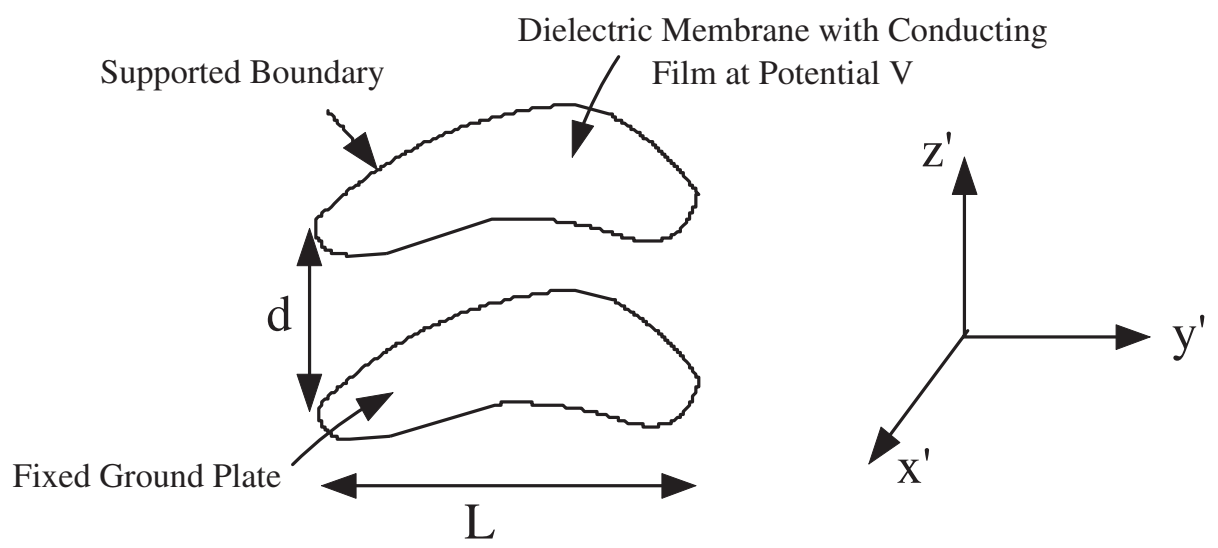

Figure 1 The simple electrostatic MEMS device.

The key component of many modern MEMS is the simple idealized electrostatic device shown in Fig. 1. The upper part of this device consists of a thin and deformable elastic membrane that is held fixed along its boundary and which lies above a rigid grounded plate. This elastic membrane is modeled as a dielectric with a small but finite thickness. The upper surface of the membrane is coated with a negligibly thin metallic conducting film. When a voltage $V$ is applied to the conducting film, the thin dielectric membrane deflects towards the bottom plate, and when $V$ is increased beyond a certain critical value $V^{*}$-known as pull-in voltagethe steady-state of the elastic membrane is lost, and proceeds to touchdown or snap through at a finite time creating the so-called pull-in instability.

A mathematical model of the physical phenomena, leading to a partial differential equation for the dimensionless dynamic deflection of the membrane, was derived and analyzed in [7] and [11]. In the damping-dominated limit, and using a narrow-gap asymptotic analysis, the dimensionless dynamic deflection $u=u(x, t)$ of the membrane on a bounded domain $\Omega$ in $\mathbb{R}^{N}$, is found to satisfy the following 
parabolic problem

$$
\begin{aligned}
& \frac{\partial u}{\partial t}=\Delta u+\frac{\lambda f(x)}{(1-u)^{2}} \quad \text { for } \quad x \in \Omega, \\
& u(x, t) \geq 0 \quad \text { for } \quad x \in \Omega \text {, } \\
& u(x, t)=0 \quad \text { for } \quad x \in \partial \Omega \text {, } \\
& u(x, 0)=0 \quad \text { for } \quad x \in \Omega .
\end{aligned}
$$

An outline of the derivation of (1.1) was given in Appendix A of [11]. This initial condition in (1.1d) assumes that the membrane is initially undeflected and the voltage is suddenly applied to the upper surface of the membrane at time $t=0$. The parameter $\lambda>0$ in (1.1a) characterizes the relative strength of the electrostatic and mechanical forces in the system, and is given in terms of the applied voltage $V$ by $\lambda=\frac{\varepsilon_{0} V^{2} L^{2}}{2 T_{e} d^{3}}$, where $d$ is the undeflected gap size (see Fig. 1 ), $L$ is the length scale of the membrane, $T_{e}$ is the tension of the membrane, and $\varepsilon_{0}$ is the permittivity of free space in the gap between the membrane and the bottom plate. We shall use here the parameter $\lambda$ (resp., $\lambda^{*}$ ) to represent the applied voltage $V$ (resp., pull-in voltage $V^{*}$ ). Referred to as the permittivity profile, $f(x)$ in $(1.1 a)$ is defined by the ratio $f(x)=\frac{\varepsilon_{0}}{\varepsilon_{2}(x)}$ where $\varepsilon_{2}(x)$ is the dielectric permittivity of the thin membrane.

There are several issues that must be considered in the actual design of MEMS devices. Typically one of the primary goals is to achieve the maximum possible stable deflection before touchdown occurs, which is referred to as pullin distance (cf. [11] and [15]). Another consideration is to increase the stable operating range of the device by improving the pull-in voltage $\lambda^{*}$ subject to the constraint that the range of applied voltage is limited by the available power supply. Such improvements in the stable operating range are important for the design of certain MEMS devices such as microresonators. One way -studied in [15] and [11]- of achieving larger values of $\lambda^{*}$, while simultaneously increasing the pullin distance, is to introduce a spatially varying dielectric permittivity $\varepsilon_{2}(x)$ of the membrane. The idea is to locate the region where the membrane deflection would normally be largest under a spatially uniform permittivity, and then make sure that a new dielectric permittivity $\varepsilon_{2}(x)$ is largest -and consequently the profile $f(x)$ smallest - in that region.

This latter approach requires the membrane having varying dielectric properties, a framework investigated recently in [15] and [11]. In [15] J. Pelesko studied the steady-states of (1.1), when $f(x)$ is assumed to be bounded away from zero. He established in this case an upper bound $\bar{\lambda}_{1}$ for $\lambda^{*}$, and derived numerical results for the power-law permittivity profile, from which the larger pull-in voltage and thereby the larger pull-in distance, the existence and multiplicity of the steady-states were observed. Recently, Y. Guo, Z. Pan and M. Ward studied in [11] the dynamic behavior of (1.1), which is also of great practical interest. They considered a more general class of profiles $f(x)$, where the membrane is allowed to be perfectly conducting, i.e., $0 \leq f(x) \leq 1$ on $\Omega$ with $f(x)>0$ on a subset 
of positive measure. By using both analytical and numerical techniques, they obtained larger pull-in voltage $\lambda^{*}$ and larger pull-in distance for different classes of varying permittivity profiles. These results were extended and sharpened in [9], where we focussed on the steady-state solutions of (1.1), i.e,

$$
\begin{array}{rlrl}
-\Delta u & =\frac{\lambda f(x)}{(1-u)^{2}} & & x \in \Omega, \\
u(x) & =0 & x \in \partial \Omega .
\end{array}
$$

with $0<u<1$ on $\Omega$, and the permittivity profile $f(x)$ was allowed to vanish somewhere and satisfy

$$
\begin{aligned}
& f \in C^{\alpha}(\bar{\Omega}) \text { for some } \alpha \in(0,1], 0 \leq f \leq 1 \text { and } \\
& f>0 \text { on a subset of } \Omega \text { of positive measure. }
\end{aligned}
$$

We establish in particular the following lower and upper bound estimates on the pull-in voltage. Here we write $|\Omega|$ for the volume of a domain $\Omega$ in $\mathbb{R}^{N}$ and $P(\Omega):=\int_{\partial \Omega} d s$ for its "perimeter", with $\omega_{N}$ referring to the volume of the unit ball $B_{1}(0)$ in $\mathbb{R}^{N}$. We denote by $\mu_{\Omega}$ the first eigenvalue of $-\Delta$ on $H_{0}^{1}(\Omega)$, and by $\phi_{\Omega}$ the corresponding positive eigenfunction normalized with $\int_{\Omega} \phi_{\Omega} d x=1$.

Theorem A (Theorem $\mathbf{1 . 1}$ in [9]) Assume $f$ is a function satisfying (1.2) on a bounded domain $\Omega$ in $\mathbb{R}^{N}$, then there exists a finite pull-in voltage $\lambda^{*}:=$ $\lambda^{*}(\Omega, f)>0$ with the following properties:

1. If $0 \leq \lambda<\lambda^{*}$, there exists at least one solution for $(S)_{\lambda}$.

2. If $\lambda>\lambda^{*}$, there is no solution for $(S)_{\lambda}$.

3. The following bounds on $\lambda^{*}$ hold for any bounded domain $\Omega$ :

$$
\begin{aligned}
& \max \left\{\frac{8 N}{27}, \frac{6 N-8}{9}\right\} \frac{1}{\sup _{\Omega} f}\left(\frac{\omega_{N}}{|\Omega|}\right)^{\frac{2}{N}} \leq \lambda^{*}(\Omega) \\
& \leq \min \left\{\bar{\lambda}_{1}:=\frac{4 \mu_{\Omega}}{27 \inf _{x \in \Omega} f(x)}, \bar{\lambda}_{2}:=\frac{\mu_{\Omega}}{3 \int_{\Omega} f \phi_{\Omega} d x}\right\} .
\end{aligned}
$$

4. If $\Omega$ is a strictly star-shaped domain with $x \cdot \nu(x) \geq a>0$ for all $x \in \partial \Omega$, where $\nu(x)$ is the unit outer normal at $x \in \partial \Omega$, and if $f \equiv 1$, then

$$
\lambda^{*}(\Omega) \leq \bar{\lambda}_{3}=\frac{(N+2)^{2} P(\Omega)}{8 a N|\Omega|} .
$$

In particular, if $\Omega=B_{1}(0) \subset \mathbb{R}^{N}$ then we have the bound $\lambda^{*}\left(B_{1}(0)\right) \leq$ $\frac{(N+2)^{2}}{8}$. 
5. If $f(x) \equiv|x|^{\alpha}$ with $\alpha \geq 0$ and $\Omega$ is a ball of radius $R$, then

$$
\begin{gathered}
\lambda^{*}\left(B_{R},|x|^{\alpha}\right) \geq \max \left\{\frac{4(2+\alpha)(N+\alpha)}{27}, \frac{(2+\alpha)(3 N+\alpha-4)}{9}\right\} R^{-(2+\alpha)} . \\
\text { Moreover, if } N \geq 8 \text { and } 0 \leq \alpha \leq \alpha^{* *}(N):=\frac{4-6 N+3 \sqrt{6}(N-2)}{4}, \text { then } \\
\qquad \lambda^{*}\left(B_{1},|x|^{\alpha}\right)=\frac{(2+\alpha)(3 N+\alpha-4)}{9} .
\end{gathered}
$$

We remark that (1) and (2) of Theorem A hold in the $H_{0}^{1}(\Omega)$-weak sense. Fine properties of steady states -such as regularity, stability, uniqueness, multiplicity, energy estimates, and comparison results- were also shown in [9] and [6] to depend on the dimension of the ambient space and on the permittivity profile. In particular, the following properties of positive minimal solutions of $(S)_{\lambda}$ were established.

Definition 1.1 A solution $u_{\lambda}$ of $(S)_{\lambda}$ is said to be a minimal solution, if $u_{\lambda}(x)$ $\leq u(x)$ in $\Omega$ whenever $u$ is any solution of $(S)_{\lambda}$. The limit of $u_{\lambda}$ as $\lambda \uparrow \lambda^{*}$ is called the extremal solution $u^{*}$ of $(S)_{\lambda}$.

For any solution $u$ of $(S)_{\lambda}$, one can introduce the linearized operator at $u$ defined by $L_{u, \lambda}=-\Delta-\frac{2 \lambda f(x)}{(1-u)^{3}}$, and its corresponding eigenvalues $\left\{\mu_{k, \lambda}(u) ; k=1,2, \ldots\right\}$. The following was also proved in [9].

Theorem B (Theorem 1.2 in [9]) Suppose $f$ is a function satisfying (1.2) on a bounded domain $\Omega$, and consider $\lambda^{*}:=\lambda^{*}(\Omega, f)$ as defined in Theorem A. Then,

1. For any $0 \leq \lambda<\lambda^{*}$, there exists a unique minimal solution $u_{\lambda}$ of $(S)_{\lambda}$ such that $\mu_{1, \lambda}\left(u_{\lambda}\right)>0$. Moreover for each $x \in \Omega$, the function $\lambda \rightarrow u_{\lambda}(x)$ is strictly increasing and differentiable on $\left(0, \lambda^{*}\right)$.

2. If $1 \leq N \leq 7$ then -by means of energy estimates- one has $\sup _{\lambda \in\left(0, \lambda^{*}\right)} \|$ $u_{\lambda} \|_{\infty}<1$ and consequently, the extremal solution $u^{*}=\lim _{\lambda \uparrow \lambda^{*}} u_{\lambda}$ exists in $C^{1, \alpha}(\bar{\Omega})$ with $0<\alpha<1$ and is a solution for $(S)_{\lambda^{*}}$ such that $\mu_{1, \lambda^{*}}\left(u^{*}\right)=0$. In particular, $u^{*}$ is unique.

3. On the other hand, if $N \geq 8, f(x)=|x|^{\alpha}$ with $0 \leq \alpha \leq \alpha^{* *}(N):=$ $\frac{4-6 N+3 \sqrt{6}(N-2)}{4}$ and $\Omega$ is the unit ball, then the extremal solution is necessarily $u^{*}(x)=1-|x|^{\frac{2+\alpha}{3}}$ and is therefore singular.

We note from [9] that in general, the extremal function $u^{*}$ exists in any dimension, does solve $(S)_{\lambda^{*}}$ in a suitable weak sense and is the unique solution in an appropriate class. The above theorem says that it is however a classical solution in dimensions $1 \leq N \leq 7$. 
In this paper, we deal with issues of global convergence as well as finite and infinite time "touchdown" in the dynamic problem (1.1). Throughout this paper and unless mentioned otherwise, solutions for (1.1) are taken in the classical sense. The permittivity profile $f(x)$ will be allowed to vanish somewhere, and will be assumed to satisfy (1.2). Recall that a point $x_{0} \in \bar{\Omega}$ is said to be a touchdown point for a solution $u(x, t)$ of $(1.1)$, if for some $T \in(0,+\infty]$, we have $\lim _{t_{n} \rightarrow T} u\left(x_{0}, t_{n}\right)=1 . T$ is then said to be a -finite or infinite- touchdown time. For each such solution, we define its corresponding - possibly infinite- "first touchdown time":

$$
T_{\lambda}(\Omega, f, u)=\inf \left\{t \in(0,+\infty] ; \sup _{x \in \bar{\Omega}} u(x, t)=1\right\} .
$$

We shall analyze the relationship between the applied voltage $\lambda$, the permittivity profile $f$, and the dynamic deflection of the elastic membrane. It is already known that solutions corresponding to large voltages $\lambda$ necessarily touchdown in finite time (See [11]). The following theorem proved in section 2, completes the picture.

Theorem 1.1 Suppose $\lambda^{*}:=\lambda^{*}(\Omega, f)$ is as in Theorem A, then the following hold:

1. If $\lambda \leq \lambda^{*}$, then there exists a unique solution $u(x, t)$ for (1.1) which globally converges as $t \rightarrow+\infty$, monotonically and pointwise to its unique minimal steady-state.

2. If $\lambda>\lambda^{*}$ and $\inf _{\Omega} f>0$, then the solution $u(x, t)$ of (1.1) must touchdown at a finite time.*

This "touchdown" phenomenon is referred to sometimes as quenching. Note that in the case where the unique minimal steady-state of (1.1) at $\lambda=\lambda^{*}$ is non-regular - which can happen if $N \geq 8$ - the above result means that the corresponding dynamic solution must touchdown but that quenching occurs here in infinite time.

In section 3 we shall establish that -an isolated- touchdown cannot occur at a point in $\Omega$ where the permittivity profile is zero, a fact that was observed numerically and conjectured to hold in [11]. More precisely, we prove the following.

Theorem 1.2 Suppose $u(x, t)$ is a touchdown solution of (1.1) at a finite time $T$, then $u_{t}>0$ for all $0<t<T$. Furthermore,

1. The permittivity profile $f$ cannot vanish on an isolated set of touchdown points in $\Omega$.

2. On the other hand, zeroes of the permittivity profile can be locations of touchdown in infinite time.

*After this paper went to press, we realized that touchdown of solutions for (1.1) does occur in finite-time when $\lambda>\lambda_{1}^{*}$ without the restriction that $\inf _{x \in \Omega} f(x)>0$. This can be done by adapting the methods of [5] (Theorem 1, p.83). 
In section 4 we shall provide upper and lower estimates for touchdown times. Uniqueness considerations lead to a first touchdown time $T_{\lambda}(\Omega, f)$ that only depend on the domain $\Omega$ and on the profile $f$. These touchdown times translate into useful information concerning the speed of the operation for many MEMS devices, such as Radio Frequency (RF) switches and microvalves. Estimates (1.9) and (1.10) below were already established in [11] for large $\lambda$. Considering that $\lambda^{*}<\min \left\{\bar{\lambda}_{1}, \bar{\lambda}_{2}\right\}$, the estimate (1.8) below gives an upper bound on the first touchdown time as soon as we exceed the pull-in voltage $\lambda^{*}$.

Theorem 1.3 Suppose $f$ is a function satisfying (1.2) on a bounded domain $\Omega$, and let $T_{\lambda}(\Omega, f)$ be the first-possibly infinite-touchdown time corresponding to a voltage $\lambda$.

1. The following lower estimate then holds for any $\lambda>0$ :

$$
\frac{1}{3 \lambda \sup _{x \in \Omega} f(x)} \leq T_{\lambda}(\Omega, f) .
$$

2. If $\inf _{x \in \Omega} f(x)>0$, then for any $\lambda>\lambda^{*}$, we have $T_{\lambda}(\Omega, f) \leq T_{0, \lambda}(\Omega, f)$ where

$$
T_{0, \lambda}(\Omega, f):=\frac{8\left(\lambda+\lambda^{*}\right)^{2}}{3\left(\lambda-\lambda^{*}\right)^{2}\left(\lambda+3 \lambda^{*}\right) \inf _{\Omega} f}\left[1+\left(\frac{\lambda+3 \lambda^{*}}{2 \lambda+2 \lambda^{*}}\right)^{1 / 2}\right] .
$$

3. If $\inf _{x \in \Omega} f(x)>0$, and $\lambda>\bar{\lambda}_{1}:=\frac{4 \mu_{\Omega}}{27 \inf _{x \in \Omega} f(x)}$, then

$$
T_{\lambda}(\Omega, f) \leq T_{1, \lambda}(\Omega, f):=\int_{0}^{1}\left[\frac{\lambda \inf _{x \in \Omega} f(x)}{(1-s)^{2}}-\mu_{\Omega} s\right]^{-1} d s .
$$

4. If $\lambda>\bar{\lambda}_{2}:=\frac{\mu_{\Omega}}{3 \int_{\Omega} f \phi_{\Omega} d x}$, then

$$
T_{\lambda}(\Omega, f) \leq T_{2, \lambda}(\Omega, f):=-\frac{1}{\mu_{\Omega}} \log \left[1-\frac{\mu_{\Omega}}{3 \lambda}\left(\int_{\Omega} f \phi_{\Omega} d x\right)^{-1}\right] .
$$

Note that the upper bounds $T_{0, \lambda}$ and $T_{1, \lambda}$ are relevant only when $f$ is bounded away from 0 , while the upper bound $T_{2, \lambda}$ is valid for all permittivity profiles provided of course that $\lambda>\bar{\lambda}_{2}$.

In a companion paper [10], the second-named author gives a refined description of the touchdown behavior of a MEMS device, including some touchdown estimates, touchdown rates, as well as some information on the location of touchdown points and on the shape of the touchdown set. 


\section{Global Convergence or Touchdown at Finite or Infinite Time}

In this section, we discuss the dynamic deflection $u=u(x, t)$ satisfying (1.1) and establish the claims in Theorem 1.1. We first prove in section $§ 2.1$ global convergence in the case $\lambda<\lambda^{*}$. In section $\S 2.2$ we study finite-time touchdown for the case $\lambda>\lambda^{*}$. Finally we discuss the case $\lambda=\lambda^{*}$ in section $\S 2.3$.

First, we note the following uniqueness result.

Lemma 2.1 Suppose $u_{1}$ and $u_{2}$ are solutions of (1.1) on the interval $[0, T]$ such that $\left\|u_{i}\right\|_{L^{\infty}(\Omega \times[0, T])}<1$ for $i=1,2$, then $u_{1}=u_{2}$.

Proof. Indeed, the difference $U=u_{1}-u_{2}$ then satisfies

$$
U_{t}-\Delta U=\alpha U \text { in } \Omega
$$

with initial data $U(x, 0)=0$ and zero boundary condition. Here

$$
\alpha(x, t)=\frac{\lambda\left(2-u_{1}-u_{2}\right) f(x)}{\left(1-u_{1}\right)^{2}\left(1-u_{2}\right)^{2}} .
$$

The assumption on $u_{1}, u_{2}$ implies that $\alpha(x, t) \in L^{\infty}(\Omega \times[0, T])$. We now fix $T_{1} \in[0, T]$ and consider the solution $\phi$ of the problem

$$
\left\{\begin{aligned}
\phi_{t}+\Delta \phi+\alpha \phi & =0 \quad x \in \Omega, 0<t<T_{1}, \\
\phi\left(x, T_{1}\right) & =\theta(x) \in C_{0}(\Omega) \\
\phi(x, t) & =0 \quad x \in \partial \Omega
\end{aligned}\right.
$$

The standard linear theory (cf. Theorem 8.1 of [14]) gives that the solution of $(2.2)$ is unique and bounded. Now multiplying (2.1) by $\phi$, and integrating it on $\Omega \times\left[0, T_{1}\right]$, together with $(2.2)$, yield that

$$
\int_{\Omega} U\left(x, T_{1}\right) \theta(x) d x=0
$$

for arbitrary $T_{1}$ and $\theta(x)$, which implies that $U \equiv 0$, and we are done.

\subsection{Global convergence when $\lambda<\lambda^{*}$}

Theorem 2.2 Suppose $\lambda^{*}:=\lambda^{*}(\Omega, f)$ is the pull-in voltage defined in Theorem $A$, then for every $\lambda<\lambda^{*}$, there exists a unique global solution $u(x, t)$ of (1.1) which monotonically converges as $t \rightarrow+\infty$ to the minimal solution $u_{\lambda}$ of $(S)_{\lambda}$.

Proof. This is standard and follows from the maximum principle combined with the existence of regular minimal steady-state solutions at this range of $\lambda$. Indeed, fix $0<\lambda<\lambda^{*}$, and use Theorem B to obtain the existence of a unique minimal 
solution $u_{\lambda}(x)$ of $(S)_{\lambda}$. It is clear that the pair $\widetilde{u} \equiv 0$ and $\widehat{u}=u_{\lambda}(x)$ are suband super-solutions of (1.1). This implies that the unique global solution $u(x, t)$ of (1.1) satisfies $1>u_{\lambda}(x) \geq u(x, t) \geq 0$ in $\Omega \times(0, \infty)$.

By differentiating in time and setting $v=u_{t}$, we get for any fixed $t_{0}>0$

$$
\begin{aligned}
v_{t} & =\Delta v+\frac{2 \lambda f(x)}{(1-u)^{3}} v & & (x, t) \in \Omega \times\left(0, t_{0}\right), \\
v(x, t) & =0 & & (x, t) \in \partial \Omega \times\left(0, t_{0}\right), \\
v(x, 0) & \geq 0 & & x \in \Omega .
\end{aligned}
$$

Here $\frac{2 \lambda f(x)}{(1-u)^{3}}$ is a locally bounded non-negative function, and by the strong maximum principle, we get that $u_{t}=v>0$ for $(x, t) \in \Omega \times\left(0, t_{0}\right)$ or $u_{t}=0$. The second case is impossible because otherwise $u(x, t)=u_{\lambda}(x)$ for any $t>0$. It follows that $u_{t}>0$ holds for all $(x, t) \in \Omega \times(0, \infty)$, and since $u(x, t)$ is bounded, this monotonicity property implies that the unique global solution $u(x, t)$ converges to some function $u_{s}(x)$ as $t \rightarrow \infty$. Hence, $1>u_{\lambda}(x) \geq u_{s}(x)>0$ in $\Omega$.

Next we claim that the limit $u_{s}(x)$ is a solution of $(S)_{\lambda}$. Indeed, consider a solution $u_{1}$ of the linear stationary boundary problem

$$
\begin{aligned}
-\Delta u_{1} & =\frac{\lambda f(x)}{\left(1-u_{s}\right)^{2}} & & x \in \Omega, \\
u_{1} & =0 & & x \in \partial \Omega .
\end{aligned}
$$

Let $w(x, t)=u(x, t)-u_{1}(x)$, then $w$ satisfies

$$
\begin{aligned}
w_{t}-\Delta w & =\lambda f(x)\left[\frac{1}{(1-u)^{2}}-\frac{1}{\left(1-u_{s}\right)^{2}}\right] \quad(x, t) \in \Omega \times(0, \infty), \\
w(x, t) & =0 \quad x \in \partial \Omega \times(0, \infty), \\
w(x, 0) & =-u_{1}(x) \quad x \in \Omega .
\end{aligned}
$$

Since the right side of $(2.8)$ converges to zero in $L^{2}(\Omega)$ as $t \rightarrow \infty$, a standard eigenfunction expansion implies that the solution $w$ of (2.8) also converges to zero in $L^{2}(\Omega)$ as $t \rightarrow \infty$. This shows that $u(x, t) \rightarrow u_{1}(x)$ in $L^{2}(\Omega)$ as $t \rightarrow \infty$. But since $u(x, t) \rightarrow u_{s}(x)$ pointwise in $\Omega$ as $t \rightarrow \infty$, we deduce that $u_{1}(x) \equiv u_{s}(x)$ in $L^{2}(\Omega)$, which implies that $u_{s}(x)$ is also a solution for $(S)_{\lambda}$. The minimal property of $u_{\lambda}(x)$ then yields that $u_{\lambda}(x) \equiv u_{s}(x)$ on $\Omega$ from which follows that for every $x \in \Omega$, we have $u(x, t) \uparrow u_{\lambda}(x)$ as $t \rightarrow \infty$.

\subsection{Touchdown at finite time when $\lambda>\lambda^{*}$}

In this case, we know from Theorem A that there is no solution for $(S)_{\lambda}$ as soon as $\lambda>\lambda^{*}$. Since the solution $u(x, t)$ of $(1.1)-$ whenever it exists - is strictly increasing in time $t$ (see preceeding theorem), then there must be $T \leq \infty$ such that $u(x, t)$ reaches 1 at some point of $\bar{\Omega}$ as $t \rightarrow T^{-}$. Otherwise, a proof similar 
to Theorem 2.2 would imply that $u(x, t)$ will converge to its steady-state which is then the unique minimal solution $u_{\lambda}$ of $(S)_{\lambda}$, contrary to the hypothesis that $\lambda>\lambda^{*}$. Therefore for this case, it only remains to know whether the touchdown time is finite or infinite. It was actually proved in [11] -via energy methods - that the touchdown time $T$ must be finite whenever $\lambda$ is large enough, but whether it is the case for any $\lambda>\lambda^{*}$ was left open. This is exactly what we prove in the following.

Theorem 2.3 Suppose $\lambda^{*}:=\lambda^{*}(\Omega, f)$ is the pull-in voltage defined in Theorem A. If $\inf _{x \in \Omega} f(x)>0$, then for $\lambda>\lambda^{*}$ there exists a finite time $T_{\lambda}(\Omega, f)$ at which the unique solution $u(x, t)$ of (1.1) must touchdown. Moreover, we have

$$
T_{\lambda}(\Omega, f) \leq T_{0, \lambda}
$$

Which is given by formula 1. Motivated by [4], we start by transforming the problem from a touchdown situation (i.e. quenching) into a blow-up problem where a concavity method can be used. For that, we set $V=1 /(1-u)$ which reduces (1.1) to the following parabolic problem

$$
\begin{aligned}
& V_{t}=\Delta V-\frac{2|\nabla V|^{2}}{V}+\lambda f(x) V^{4} \quad \text { for } \quad x \in \Omega, \\
& V(x, t)=1 \quad \text { for } x \in \partial \Omega \text {, } \\
& V(x, 0)=1 \quad \text { for } x \in \Omega \text {. }
\end{aligned}
$$

This transformation implies that when $\lambda>\lambda^{*}$, the solution of (2.12) must blow up (in finite or infinite time) and that there is no solution for the corresponding stationary equation:

$$
\Delta V-\frac{2|\nabla V|^{2}}{V}+\lambda f(x) V^{4}=0, \quad x \in \Omega ; \quad V=1, \quad x \in \partial \Omega .
$$

Therefore, proving finite touchdown time of $u$ for (1.1) is equivalent to showing finite blow-up time of the solution $V$ for (2.12).

For the proof, we shall first analyze the following auxiliary parabolic equation

$$
\begin{aligned}
& v_{t}=\Delta v-\frac{2|\nabla v|^{2}}{v}+\lambda a^{2} t^{2} f(x) v^{4} \quad \text { for } \quad x \in \Omega, \\
& v=1 \quad \text { for } x \in \partial \Omega \text {, } \\
& v(x, 0)=1 \quad \text { for } x \in \Omega \text {, }
\end{aligned}
$$

where $a>0$ is a given constant.

Lemma 2.4 Suppose $v$ is a solution of (2.14) up to a finite time $\bar{T}$, then $\left(\frac{v_{t}}{v^{4}}\right)_{t} \geq 0$ for all $t<\bar{T}$. 
Proof. Dividing (2.14a) by $v^{4}$, we obtain

$$
\frac{v_{t}}{v^{4}}=\frac{\Delta v}{v^{4}}-\frac{2|\nabla v|^{2}}{v^{5}}+\lambda a^{2} t^{2} f(x) .
$$

Setting $w=v^{-3}$, then direct calculations show that

$$
w_{t}-\Delta w+\frac{2|\nabla w|^{2}}{3 w}+3 \lambda a^{2} t^{2} f(x)=0
$$

Differentiate (2.15) twice with respect to $t$, we obtain

$$
\begin{aligned}
\left(\frac{|\nabla w|^{2}}{w}\right)_{t t} & =\left(\frac{2 \nabla w \nabla w_{t}}{w}-\frac{|\nabla w|^{2} w_{t}}{w^{2}}\right)_{t} \\
& =\frac{2 \nabla w \nabla w_{t t}}{w}+\frac{2\left|\nabla w_{t}\right|^{2}}{w}-\frac{4 \nabla w \nabla w_{t} w_{t}}{w^{2}}-\frac{|\nabla w|^{2} w_{t t}}{w^{2}}+\frac{2|\nabla w|^{2} w_{t}^{2}}{w^{3}},
\end{aligned}
$$

which means that the function

$$
z=w_{t t}=-3\left(\frac{v_{t}}{v^{4}}\right)_{t}
$$

satisfies

$$
\begin{aligned}
L(z): & =z_{t}-\Delta z+\frac{4 \nabla w}{3 w} \nabla z-\frac{2|\nabla w|^{2}}{3 w^{2}} z \\
& =-6 \lambda a^{2} f(x)-\frac{2}{3}\left[\frac{2\left|\nabla w_{t}\right|^{2}}{w}+\frac{2|\nabla w|^{2} w_{t}^{2}}{w^{3}}-\frac{4 \nabla w \nabla w_{t} w_{t}}{w^{2}}\right] \\
& \leq-6 \lambda a^{2} f(x)
\end{aligned}
$$

after an application of Cauchy-Schwarz inequality. Hence we have

$$
L(z) \leq-6 \lambda a^{2} f(x) \leq 0 .
$$

Now from (2.14) and the definition of $z$, we have $z(x, 0)=0$ and $z=0$ on $\partial \Omega$. Since the coefficients of $L$ remain bounded as long as $v$ is bounded, we conclude that $z(x, t) \leq 0$ holds for all $t<\bar{T}$. This completes the proof of Lemma 2.4.

Proof of Theorem 2.3: Let $\lambda>\lambda^{*}, \lambda^{\prime}=\lambda-\lambda^{*}>0, \delta=\inf _{x \in \Omega} f(x)>0$,

$$
a_{0}=\frac{3 \delta \lambda^{\prime}\left(4 \lambda^{*}+\lambda^{\prime}\right)}{4\left(2 \lambda^{*}+\lambda^{\prime}\right)}\left[1-\left(\frac{4 \lambda^{*}+\lambda^{\prime}}{2\left(2 \lambda^{*}+\lambda^{\prime}\right)}\right)^{1 / 2}\right]
$$

and

$$
T_{0, \lambda}=\frac{1}{a_{0}}=\frac{8\left(\lambda+\lambda^{*}\right)^{2}}{3 \delta\left(\lambda-\lambda^{*}\right)^{2}\left(\lambda+3 \lambda^{*}\right)}\left[1+\left(\frac{\lambda+3 \lambda^{*}}{2 \lambda+2 \lambda^{*}}\right)^{1 / 2}\right]<+\infty .
$$


Consider now a solution $v$ of (2.14) corresponding to $\lambda=\lambda^{*}+\lambda^{\prime}$ and $a_{0}$ as defined in (2.18a). We first establish the following

Claim: There exists $x_{0} \in \Omega$ such that $v\left(x_{0}, t\right) \rightarrow \infty$ as $t \nearrow T_{0, \lambda}$.

Indeed, let $t_{0}=\frac{1}{a_{0}}\left[\frac{4 \lambda^{*}+\lambda^{\prime}}{2\left(2 \lambda^{*}+\lambda^{\prime}\right)}\right]^{1 / 2}$ in such a way that

$$
t_{0}<T_{0, \lambda} \quad \text { and } \quad a_{0}^{2} t_{0}^{2}\left(\lambda^{*}+\frac{\lambda^{\prime}}{2}\right)=\lambda^{*}+\frac{\lambda^{\prime}}{4} .
$$

We claim that there exists $x_{0} \in \Omega$ such that

$$
\Delta v\left(x_{0}, t_{0}\right)-\frac{2\left|\nabla v\left(x_{0}, t_{0}\right)\right|^{2}}{v\left(x_{0}, t_{0}\right)}+\left(\lambda^{*}+\frac{\lambda^{\prime}}{4}\right) f\left(x_{0}\right)\left|v\left(x_{0}, t_{0}\right)\right|^{4}>0 .
$$

Essentially, otherwise we get that for all $x \in \Omega$

$$
\Delta v\left(x, t_{0}\right)-\frac{2\left|\nabla v\left(x, t_{0}\right)\right|^{2}}{v\left(x, t_{0}\right)}+\left(\lambda^{*}+\frac{\lambda^{\prime}}{4}\right) f(x)\left|v\left(x, t_{0}\right)\right|^{4} \leq 0 .
$$

Since $v\left(x, t_{0}\right) \geq 1$ on $\Omega$, this means that the function $\bar{v}(x)=v\left(x, t_{0}\right)$ is a supersolution for the equation

$$
\Delta V-\frac{2|\nabla V|^{2}}{V}+\left(\lambda^{*}+\frac{\lambda}{4}\right) f(x) V^{4}=0, \quad x \in \Omega ; \quad V=1, \quad x \in \partial \Omega .
$$

Since $\underline{v} \equiv 1$ is obviously a subsolution of $(2.21)$, it follows that the latter has a solution which contradicts the fact that $\lambda=\lambda^{*}+\frac{\lambda^{\prime}}{4}>\lambda^{*}(f, \Omega)$. Hence, assertion (2.19) is verified.

On the other hand, we do get from (2.14) that for $t=t_{0}$ and every $x \in \Omega$,

$$
v_{t}=\Delta v-\frac{2|\nabla v|^{2}}{v}+\left(\lambda^{*}+\frac{\lambda^{\prime}}{4}\right) f(x) v^{4}+\frac{\lambda^{\prime}}{2} a_{0}^{2} t_{0}^{2} f(x) v^{4} .
$$

We then deduce from $(2.22)$ and $(2.19)$ that at the point $\left(x_{0}, t_{0}\right)$, we have

$$
\frac{v_{t}}{v^{4}} \geq \frac{\lambda^{\prime}}{2} a_{0}^{2} t_{0}^{2} f\left(x_{0}\right)>0
$$

Applying Lemma 2.4, we then get for all $\left(x_{0}, t\right), t_{0} \leq t<T_{0, \lambda}$ that:

$$
\frac{v_{t}}{v^{4}} \geq \frac{\lambda^{\prime}}{2} a_{0}^{2} t_{0}^{2} f\left(x_{0}\right)>0 .
$$

Integrating (2.23) with respect to $t$ in $\left(t_{0}, T_{0, \lambda}\right)$, we obtain since $f\left(x_{0}\right) \geq \delta$ that:

$$
\frac{1}{3}\left(1-v^{-3}\left(x_{0}, T_{0, \lambda}\right)\right) \geq \frac{\lambda^{\prime}}{2} a_{0}^{2} t_{0}^{2} f\left(x_{0}\right)\left(T_{0, \lambda}-t_{0}\right) \geq \frac{\lambda^{\prime}}{2} a_{0}^{2} t_{0}^{2} \delta\left(T_{0, \lambda}-t_{0}\right)=\frac{1}{3} .
$$

It follows that $v\left(x_{0}, t\right) \rightarrow \infty$ as $t \nearrow T_{0, \lambda}$, and the claim is proved. 
To complete the proof of Theorem 2.3, we note that since $a_{0}^{2} t^{2} \leq 1$ for all $t \leq T_{0, \lambda}$, we get from (2.14) that

$$
v_{t} \leq \Delta v-\frac{2|\nabla v|^{2}}{v}+\lambda f(x) v^{4}, \quad(x, t) \in \Omega \times\left(0, T_{0, \lambda}\right) .
$$

Setting $w=V-v$, where $V$ is the solution of (2.12), then $w$ satisfies

$$
\begin{aligned}
w_{t}-\Delta w & -\frac{2 \nabla(V+v)}{V} \\
\nabla w & +\left[\lambda\left(V^{2}+v^{2}\right)(V+v) f(x)+\frac{2|\nabla v|^{2}}{V v}\right] w \geq 0, \quad(x, t) \in \Omega \times\left(0, T_{0, \lambda}\right) .
\end{aligned}
$$

Here the coefficients of $\nabla w$ and $w$ are bounded functions as long as $V$ and $v$ are both bounded. It is also clear that $w=0$ on $\partial \Omega$ and $w(x, 0)=0$. Applying the maximum principle, we reduce that $w \geq 0$ and thus $V \geq v$. Consequently, $V$ must also blow up at some finite time $T \leq T_{0, \lambda}$, which means that $u$ must touchdown at some finite time prior to $T_{0, \lambda}$.

\subsection{Global convergence or touchdown in infinite time for $\lambda=\lambda^{*}$}

We now discuss the dynamic behavior of (1.1) at $\lambda=\lambda^{*}$. For this critical case, there exists a unique steady-state $w^{*}$ of (1.1) obtained as a pointwise limit of the minimal solution $u_{\lambda}$ as $\lambda \uparrow \lambda^{*}$. If $w^{*}$ is regular (i.e, if it is a classical solution such as in the case when $N \leq 7$ ) a similar proof as in the case where $\lambda<\lambda^{*}$, yields the existence of a unique solution $u^{*}(x, t)$ which globally converges to the unique steady-state $w^{*}$ as $t \rightarrow \infty$. On the other hand, if $w^{*}$ is a non-regular steadystate, i.e. if $\left\|w^{*}\right\|_{\infty}=1$, the situation is complicated as we shall still prove global convergence to the extremal solution $w^{*}$, which then amounts to a touchdown in infinite time.

Throughout this subsection, we shall consider the unique solution $0 \leq u^{*}=$ $u^{*}(x, t)<1$ for the problem

$$
\begin{aligned}
& u_{t}^{*}-\Delta u^{*}=\frac{\lambda^{*} f(x)}{\left(1-u^{*}\right)^{2}} \quad \text { for } \quad(x, t) \in \Omega \times\left[0, t^{*}\right), \\
& u^{*}(x, t)=0 \quad \text { for } \quad x \in \partial \Omega \times\left[0, t^{*}\right) \text {, } \\
& u^{*}(x, 0)=0 \quad \text { for } \quad x \in \Omega \text {, }
\end{aligned}
$$

where $t^{*}$ is the maximal time for existence. We shall use techniques developed in [5] to establish the following

Theorem 2.5 If $w^{*}$ is a non-regular minimal steady-state of (2.24), then there exists a unique global solution $u^{*}$ of $(2.24)$ such that $u^{*}(x, t) \leq w^{*}(x)$ for all $t<\infty$, while $u^{*}(x, t) \rightarrow w^{*}(x)$ as $t \rightarrow \infty$. In particular, $\lim _{t \rightarrow+\infty}\left\|u^{*}(x, t)\right\|_{\infty}=1$.

We shall use the following fact which is essentially Lemma 7 of [5]. 
Lemma 2.6 Consider the function $\delta(x):=\operatorname{dist}(x, \partial \Omega)$, then for any $0<T<\infty$, there exists $\varepsilon_{1}=\varepsilon_{1}(T)$ such that for $0<\varepsilon \leq \varepsilon_{1}$ the solution $Z^{\epsilon}$ of the problem

$$
\begin{aligned}
Z_{t}-\Delta Z & =-\varepsilon f(x) & \text { in } \quad \Omega \times(0, \infty), \\
Z(x, t) & =0 & \text { on } \partial \Omega \times(0, \infty), \\
Z(x, 0) & =\delta(x) & \text { in } \Omega
\end{aligned}
$$

satisfies $Z^{\epsilon} \geq 0$ on $[0, T] \times \bar{\Omega}$.

Proof of Theorem 2.5: We proceed in four steps.

Claim 1. We have that $u^{*}(x, t) \leq w^{*}(x)$ for all $(x, t) \in \Omega \times\left[0, t^{*}\right)$. Indeed, fix any $T<t^{*}$ and let $\xi$ be the solution of the backward heat equation:

$$
\begin{aligned}
\xi_{t}-\Delta \xi & =h(x, t) \quad \text { in } \quad \Omega \times(0, T), \\
\left.\xi\right|_{\partial \Omega} & =0, \quad \xi(T)=0,
\end{aligned}
$$

where $h(x, t) \geq 0$ is in $\Omega \times(0, T)$. Multiplying (2.24) by $\xi$ and integrating on $\Omega \times(0, T)$ we find that

$$
\int_{0}^{T} \int_{\Omega} u^{*} h d x d t=\int_{0}^{T} \int_{\Omega} \frac{\lambda^{*} \xi f(x)}{\left(1-u^{*}\right)^{2}} d x d t .
$$

On the other hand,

$$
\begin{aligned}
-\int_{0}^{T} \int_{\Omega} w^{*} \xi_{t} d x d t & =\int_{\Omega} w^{*} \xi(0) d x \\
-\int_{0}^{T} \int_{\Omega} w^{*} \Delta \xi d x d t & =\int_{0}^{T} \int_{\Omega} \frac{\lambda^{*} \xi f(x)}{\left(1-w^{*}\right)^{2}} d x d t .
\end{aligned}
$$

Therefore, we have

$$
\begin{aligned}
\int_{0}^{T} \int_{\Omega}\left(u^{*}-w^{*}\right) h d x d t & \leq \int_{\Omega} w^{*} \xi(0) d x+\int_{0}^{T} \int_{\Omega}\left(u^{*}-w^{*}\right) h d x d t \\
& =\int_{0}^{T} \int_{\Omega}\left(\frac{1}{\left(1-u^{*}\right)^{2}}-\frac{1}{\left(1-w^{*}\right)^{2}}\right) \lambda^{*} \xi f(x) d x d t \\
& \leq C \int_{0}^{T} \int_{\left\{u^{*} \geq w^{*}\right\}}\left(\frac{1}{\left(1-u^{*}\right)^{2}}-\frac{1}{\left(1-w^{*}\right)^{2}}\right) \xi d x d t \\
& \leq C \int_{0}^{T} \int_{\Omega}\left(u^{*}-w^{*}\right)^{+} \xi d x d t
\end{aligned}
$$

since $\left\|u^{*}\right\|_{\infty}<1$ for $t \in[0, T)$. Therefore, we have

$$
\int_{0}^{T} \int_{\Omega}\left(u^{*}-w^{*}\right) h d x d t \leq C\left(\int_{0}^{T} \int_{\Omega}\left[\left(u^{*}-w^{*}\right)^{+}\right]^{2} d x d t\right)^{1 / 2}\left(\int_{0}^{T} \int_{\Omega} \xi^{2} d x d t\right)^{1 / 2} .
$$


On the other hand, $\xi(x, t)=\int_{t}^{T} T(s-t) h(x, s) d s$, where $T(t)$ is the heat semigroup with Dirichlet boundary condition, and hence

$$
\|\xi(x, t)\|_{L^{2}}^{2} \leq\left(\int_{t}^{T}\|h(x, s)\|_{L^{2}} d s\right)^{2} \leq(T-t) \int_{0}^{T} \int_{\Omega} h^{2} d x d t .
$$

Therefore,

$$
\int_{0}^{T} \int_{\Omega} \xi^{2} d x d t \leq \frac{T^{2}}{2} \int_{0}^{T} \int_{\Omega} h^{2} d x d t
$$

and so,

$$
\int_{0}^{T} \int_{\Omega}\left(u^{*}-w^{*}\right) h d x d t \leq \frac{C T}{\sqrt{2}}\left(\int_{0}^{T} \int_{\Omega}\left[\left(u^{*}-w^{*}\right)^{+}\right]^{2} d x d t\right)^{1 / 2}\left(\int_{0}^{T} \int_{\Omega} h^{2} d x d t\right)^{1 / 2}
$$

Letting $h$ converge to $\left(u^{*}-w^{*}\right)^{+}$in $L^{2}$, and since $u^{*}-w^{*} \in L^{1}(\Omega)$ we have

$$
\int_{0}^{T} \int_{\Omega}\left[\left(u^{*}-w^{*}\right)^{+}\right]^{2} d x d t \leq \frac{C T}{\sqrt{2}} \int_{0}^{T} \int_{\Omega}\left[\left(u^{*}-w^{*}\right)^{+}\right]^{2} d x d t
$$

which gives that $u^{*} \leq w^{*}$ provided $C^{2} T^{2}<2$, and our first claim follows.

Claim 2. There exist $0<\tau_{1}<t^{*}$, and $C_{0}, c_{0}>0$ such that for all $x \in \Omega$

$$
u^{*}\left(x, \tau_{1}\right) \leq \min \left\{C_{0} \delta(x) ; w^{*}(x)-c_{0} \delta(x)\right\} .
$$

Fix $0<\tau<t^{*}$ sufficiently small, and let $v$ be the solution of

$$
\begin{aligned}
& v_{t}-\Delta v=\frac{\lambda^{*} f(x)}{(1-v)^{2}} \quad \text { for } \quad(x, t) \in \Omega \times[0, \bar{T}), \\
& v(x, t)=0 \quad \text { for } \quad x \in \partial \Omega \times[0, \bar{T}), \\
& v(x, 0)=v_{0}=u^{*}(x, \tau) \quad \text { for } \quad x \in \Omega,
\end{aligned}
$$

where $[0, \bar{T})$ is the maximal interval of existence for $v$. Similarly to Claim 1 , we can show that $0 \leq v \leq w^{*}$. Choose now $K>1$ sufficiently large such that the path $z(x, t):=u^{*}(x, t)+\frac{1}{K} T(t) v_{0}$ satisfies $\|z(x, t)\|_{\infty} \leq 1$ for $0 \leq t<\bar{T}$. We then have

$$
\begin{aligned}
z_{t}-\Delta z & =\frac{\lambda^{*} f(x)}{\left(1-u^{*}\right)^{2}} \leq \frac{\lambda^{*} f(x)}{(1-z)^{2}} & & \text { in } \quad \Omega \times(0, \bar{T}), \\
z(x, t) & =0 & & \text { on } \quad \partial \Omega \times(0, \bar{T}), \\
z(x, 0) & =\frac{v_{0}(x)}{K} & & \text { in } \quad \Omega,
\end{aligned}
$$

and the maximum principle gives that $z \leq v$. Consider now a function $\gamma:[0, \infty) \rightarrow$ $R$ such that $\gamma(t)>0$ and

$$
T(t) v_{0} \geq K \gamma(t) \delta \text { on } \Omega
$$


We then get

$$
u^{*} \leq v-\frac{1}{K} T(t) v_{0} \leq w^{*}-\frac{1}{K} T(t) v_{0} \leq w^{*}-\gamma(t) \delta \quad \text { for } \quad 0 \leq t<\bar{T} .
$$

Consider now the solution $\xi_{0}$

$$
-\Delta \xi_{0}=1 \quad \text { in } \Omega ; \quad \xi_{0}=0 \quad \text { on } \quad \partial \Omega
$$

in such a way that $\xi_{0}=T(t) \xi_{0}+\int_{0}^{t} T(s) 1_{\Omega} d s$ for all $0 \leq t \leq T<\min \left\{\bar{T}, t^{*}\right\}$. Since $T(t) \xi_{0} \geq 0$ it follows that $\int_{0}^{t} T(s) 1_{\Omega} d s \leq \xi_{0} \leq C \delta$. On the other hand, for any $0 \leq t \leq T<t^{*}$, $u^{*}$ is bounded by some constant $M<1$ on $\bar{\Omega} \times[0, T]$ such that

$$
u^{*} \leq M T(t) 1_{\Omega}+\frac{C}{(1-M)^{2}} \int_{0}^{t} T(s) 1_{\Omega} d s .
$$

Consider now a function $C:[0, \infty) \rightarrow R$ such that $T(t) 1_{\Omega} \leq C(t) \delta$ for $t \geq 0$, which means that

$$
u^{*} \leq M C(t) \delta+C(M) C \delta
$$

for any $0 \leq t \leq T$. This combined with (2.28) concludes the proof of Claim (2.25).

Claim 3. For $0<\varepsilon<1$, there exists $w_{\varepsilon}$ satisfying $\left\|w_{\varepsilon}\right\|_{\infty}<1$ and

$$
\int_{\Omega} \nabla w_{\varepsilon} \nabla \varphi \geq \int_{\Omega}\left(\frac{1}{\left(1-w_{\varepsilon}\right)^{2}}-\varepsilon\right) \lambda^{*} \varphi f(x)
$$

for all $\varphi \in H_{0}^{1}(\Omega)$ with $\varphi \geq 0$ on $\Omega$. Moreover, there exists $0<\varepsilon_{1} \leq 1$ such that for $0<\varepsilon<\varepsilon_{1}$, we also have

$$
0 \leq w_{\varepsilon}(x)-\frac{c_{0}}{2} \delta(x) \quad \text { for } x \in \Omega
$$

$c_{0}$ being as in (2.25).

To prove (2.29), we set

$$
g\left(w^{*}\right)=\frac{1}{\left(1-w^{*}\right)^{2}}, \quad h\left(w^{*}\right)=\int_{0}^{w^{*}} \frac{d s}{g(s)}, \quad 0 \leq w^{*}<1 .
$$

For any $\varepsilon \in(0,1)$ we also set

$$
\widetilde{g}\left(w^{*}\right)=\frac{1}{\left(1-w^{*}\right)^{2}}-\varepsilon, \quad \widetilde{h}\left(w^{*}\right)=\int_{0}^{w^{*}} \frac{d s}{\widetilde{g}(s)}, \quad 0 \leq w^{*}<1,
$$

and $\phi_{\varepsilon}\left(w^{*}\right):=\widetilde{h}^{-1}\left(h\left(w^{*}\right)\right)$. It is easy to check that $\phi_{\varepsilon}(0)=0$ and $0 \leq \phi_{\varepsilon}(s)<s$ for $s \geq 0$, and $\phi_{\varepsilon}$ is increasing and concave with

$$
\phi_{\varepsilon}^{\prime}(s)=\frac{g\left(\phi_{\varepsilon}(s)\right)-\varepsilon}{g(s)}>0 .
$$


Setting $w_{\varepsilon}=\phi_{\varepsilon}\left(w^{*}\right)$, we have for any $\varphi \in H_{0}^{1}(\Omega)$ with $\varphi \geq 0$ on $\Omega$,

$$
\begin{aligned}
\int_{\Omega} \nabla w_{\varepsilon} \nabla \varphi & =\int_{\Omega} \phi_{\varepsilon}^{\prime}\left(w^{*}\right) \nabla w^{*} \nabla \varphi=\int_{\Omega} \nabla w^{*} \nabla\left(\phi_{\varepsilon}^{\prime}\left(w^{*}\right) \varphi\right)-\int_{\Omega} \phi_{\varepsilon}^{\prime \prime}\left(w^{*}\right) \varphi\left|\nabla w^{*}\right|^{2} \\
& \geq \int_{\Omega} \frac{\lambda^{*} f(x)}{\left(1-w^{*}\right)^{2}} \phi_{\varepsilon}^{\prime}\left(w^{*}\right) \varphi=\int_{\Omega}\left(\frac{1}{\left(1-w_{\varepsilon}\right)^{2}}-\varepsilon\right) \lambda^{*} \varphi f(x),
\end{aligned}
$$

which gives $(2.29)$ for any $\varepsilon \in(0,1)$.

In order to prove $(2.30)$, we set

$$
\eta(x)=\min \left\{w^{*}(x),\left(C_{0}+c_{0}\right) \delta(x)\right\} \quad \text { and } \quad \eta_{\varepsilon}=\phi_{\varepsilon} \circ \eta,
$$

where $\phi_{\varepsilon}(\cdot)$ is defined above, and $C_{0}$ and $c_{0}$ are as in (2.25). Since $\eta \leq w^{*}$ and $\phi_{\varepsilon}$ is increasing, we have $\eta_{\varepsilon} \leq \phi_{\varepsilon}\left(w^{*}\right)=w_{\varepsilon}$. Applying (2.25) we get that

$$
0 \leq \eta(x)-c_{0} \delta(x) \text { on } \Omega \text {. }
$$

We also note that $\eta_{\varepsilon}=\phi_{\varepsilon}(\eta) \leq \eta \leq M$ with $M=\left(C_{0}+c_{0}\right) \delta(x)$, and $\phi_{\varepsilon}^{\prime}(s) \rightarrow 1$ as $\varepsilon \rightarrow 0$ uniformly in $[0,1]$. Therefore, for some $\theta \in(0,1)$ we have

$$
\begin{aligned}
\eta-\eta_{\varepsilon} & =\eta-\left(\phi_{\varepsilon}(\eta)-\phi_{\varepsilon}(0)\right)=\eta\left(1-\phi_{\varepsilon}^{\prime}(\theta \eta)\right) \leq \eta \sup _{\{0 \leq s \leq 1\}}\left(1-\phi_{\varepsilon}^{\prime}(s)\right) \\
& \leq\left(C_{0}+c_{0}\right) \delta \sup _{\{0 \leq s \leq 1\}}\left(1-\phi_{\varepsilon}^{\prime}(s)\right) \leq \frac{c_{0}}{2} \delta
\end{aligned}
$$

provided $\varepsilon$ small enough, which gives

$$
\eta \leq \eta_{\varepsilon}+\frac{c_{0}}{2} \delta
$$

We now conclude from (2.33) and (2.34) that

$$
0 \leq \eta-c_{0} \delta \leq \eta_{\varepsilon}-\frac{c_{0}}{2} \delta \leq w_{\varepsilon}-\frac{c_{0}}{2} \delta
$$

for small $\varepsilon>0$, and (2.30) is therefore proved.

To complete the proof of Theorem 2.5, we assume that $t^{*}<\infty$ and we shall work towards a contradiction. In view of Claim 3), we let $\varepsilon>0$ be small enough so that $0 \leq w_{\varepsilon}-\frac{c_{0}}{2} \delta$, and use Lemma 2.6 to choose $k=k(\in) \geq 2$ such that the solution $Z$ of the problem

$$
\begin{aligned}
Z_{t}-\Delta Z & =-\varepsilon \lambda^{*} f(x) & & \text { in } \Omega \times\left(0, t^{*}\right), \\
Z(x, t) & =0 & & \text { on } \quad \partial \Omega \times\left(0, t^{*}\right), \\
Z(x, 0) & =\frac{c_{0}}{K} \delta & & \text { in } \quad \Omega
\end{aligned}
$$

satisfies $0 \leq Z<1-u^{*}$ on $\bar{\Omega} \times\left(0, t^{*}\right)$. Let $v$ be the solution of

$$
\begin{aligned}
v_{t}-\Delta v & =\left(\frac{1}{(1-|v|)^{2}}-\varepsilon\right) \lambda^{*} f(x) & & \text { in } \Omega \times\left(0, s^{*}\right), \\
v(x, t) & =0 & & \text { on } \partial \Omega \times\left(0, s^{*}\right), \\
v(x, 0) & =w_{\varepsilon} & & \text { in } \Omega,
\end{aligned}
$$


where $\left[0, s^{*}\right)$ is the maximal interval of existence for $v$. Setting $z(x, t)=Z(x, t)+$ $u^{*}(x, t)$ for $0 \leq t<t^{*}$, we then have $0 \leq u^{*} \leq z<1$ and

$$
\begin{aligned}
& z_{t}-\Delta z=\left(\frac{1}{\left(1-u^{*}\right)^{2}}-\varepsilon\right) \lambda^{*} f(x) \leq\left(\frac{1}{(1-z)^{2}}-\varepsilon\right) \lambda^{*} f(x) \quad \text { in } \quad \Omega \times\left(0, t^{*}\right), \\
& z(x, t)=0 \quad \text { on } \partial \Omega \times\left(0, t^{*}\right), \\
& z(x, 0)=\frac{c_{0}}{K} \delta(x) \leq w_{\varepsilon}(x) \quad \text { in } \quad \Omega .
\end{aligned}
$$

Now the maximum principle gives that $z \leq v$ on $\Omega \times\left(0, \min \left\{s^{*}, t^{*}\right\}\right)$, and in particular we have $0 \leq v$ on $\Omega \times\left(0, \min \left\{s^{*}, t^{*}\right\}\right)$. Furthermore, the maximum principle and (2.29) also yield that $v \leq w_{\varepsilon}$. Since $\left\|w_{\varepsilon}\right\|_{\infty}<1$ we necessarily have $t^{*}<s^{*}=\infty$. Therefore, $u^{*} \leq z \leq v \leq w_{\varepsilon}$ on $\left[0, t^{*}\right)$, which implies that $\left\|u^{*}\right\|_{\infty}<1$ at $t=t^{*}$, which contradicts to our initial assumption that $u^{*}$ is not a regular solution.

\section{The location of touchdown points}

We first present a couple of numerical simulations for different domains, different permittivity profiles, and various values of $\lambda$, by applying an implicit CrankNicholson scheme (see [11] for details), on the problem

$$
\begin{aligned}
& \frac{\partial u}{\partial t}=\Delta u-\frac{\lambda f(x)}{(1+u)^{2}} \quad \text { for } \quad x \in \Omega, \\
& u(x, t)=0 \quad \text { for } \quad x \in \partial \Omega, \\
& u(x, 0)=0 \quad \text { for } \quad x \in \Omega,
\end{aligned}
$$

in the following two choices for the domain $\Omega$

$$
\Omega:[-1 / 2,1 / 2] \quad \text { (Slab); } \quad \Omega: x^{2}+y^{2} \leq 1 \quad \text { (Unit Disk). }
$$

Simulation 1: We consider $f(x)=|2 x|$ for a permittivity profile in the slab domain $-1 / 2 \leq x \leq 1 / 2$. Here the number of the meshpoints is chosen as $N=2000$ for the plots $u$ versus $x$ at different times. Fig. 2(a) shows, for $\lambda=4.38$, a typical sequence of solutions $u$ for (3.1) approaching to the maximal negative steady-state. In Fig. 2(b) we take $\lambda=4.50$, and a touchdown behavior is observed at two different nonzero points $x= \pm 0.14132$. These numerical results and Theorem 1.1 point to a pull-in voltage $4.38 \leq \lambda^{*}<4.50$.

Simulation 2: Here we consider $f(r)=r$ for a permittivity profile in the unit disk domain. The number of meshpoints is again chosen to be $N=2000$ for the plots $u$ versus $r$ at different times. Fig. 3(a) shows how for $\lambda=1.70$, a typical sequence of solutions $u$ for (3.1) approach to the maximal negative steadystate. In Fig. 3(b) we take $\lambda=1.80$ and a touchdown behavior is observed at the nonzero points $r=0.21361$. Again these numerical results point to a pull-in voltage $1.70 \leq \lambda^{*}<1.80$. 

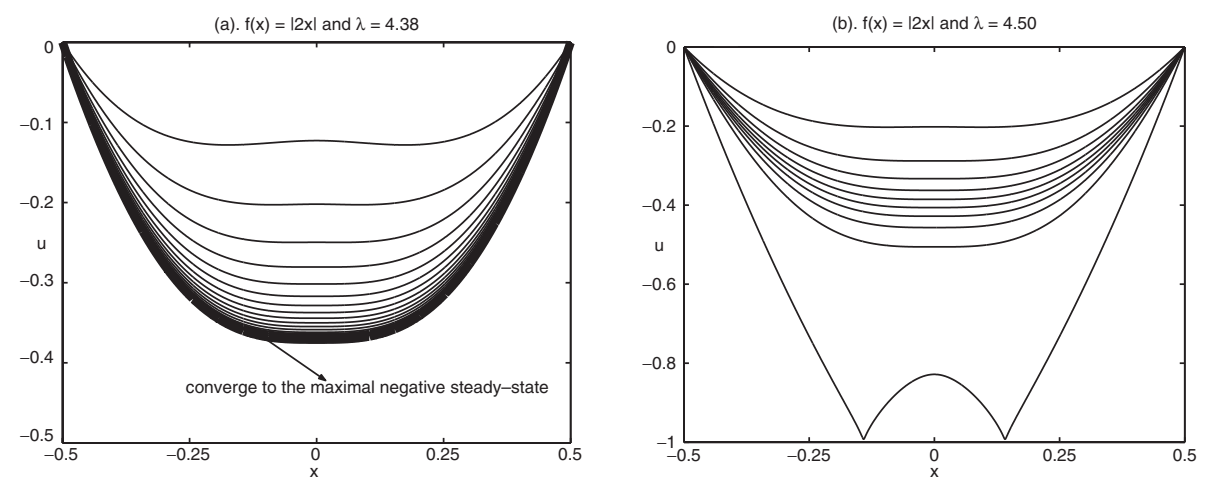

Figure 2 Left figure: for $\lambda=4.38$ we plot $u$ versus $x$ at different times showing the approach to the maximal negative steady-state. Right figure: for $\lambda=4.50$ we plot $u$ versus $x$ at different times $t=0,0.1880,0.3760,0.5639,0.7519,0.9399,1.1279,1.3159,1.5039,1.6918,1.879818$, from which touchdown is observed at $x= \pm 0.14132$. For both cases, we consider (3.1) with $f(x)=|2 x|$ in the slab domain

One can note that touchdown points at finite time are not the zero points of the varying permittivity profile $f$, a fact already observed and conjectured in [11]. Here we give a proof for this interesting phenomenon also stated in Theorem 1.2 of the introduction.

Theorem 3.1 Let $T$ be the first touchdown time for a solution $u(x, t)$ of (1.1). If $T$ is finite, then $u_{t}>0$ for all $0<t<T$. Moreover, if $K$ is an isolated set of touchdown points in $\Omega$, then necessarily $\inf _{x \in K} f(x)>0$.

On the other hand, (1.1) can have solutions that touchdown in infinite time at points $x \in \Omega$ where $f(x)=0$.

The proof of Theorem 3.1 is based on the following Harnack-type estimate.

Lemma 3.2 For any compact subset $K$ of $\Omega$ and any $m>0$, there exists a constant $C=C(K, m)>0$ such that $\sup _{x \in K}|u(x)| \leq C<1$ whenever $u$ satisfies

$$
\begin{array}{cc}
\Delta u \geq \frac{m}{(1-u)^{2}} & x \in \Omega, \\
0 \leq u<1 & x \in \Omega .
\end{array}
$$

Proof. Setting $v=1 /(1-u)$, then $(3.3)$ gives that $v$ satisfies

$$
\frac{\Delta v}{v^{2}}-\frac{2|\nabla v|^{2}}{v^{3}} \geq m v^{2} \quad \text { in } \Omega,
$$

which means that $v$ is a subsolution of the "linear" equation $\Delta v=0$ in $\Omega$. In order to apply the Harnack inequality on $v$, we need to show that for balls $B_{r} \subset \Omega$, we have that $v \in L^{3}\left(B_{r}\right)$ with an $L^{3}$-norm that only depends on $m$ and the radius $r$. 

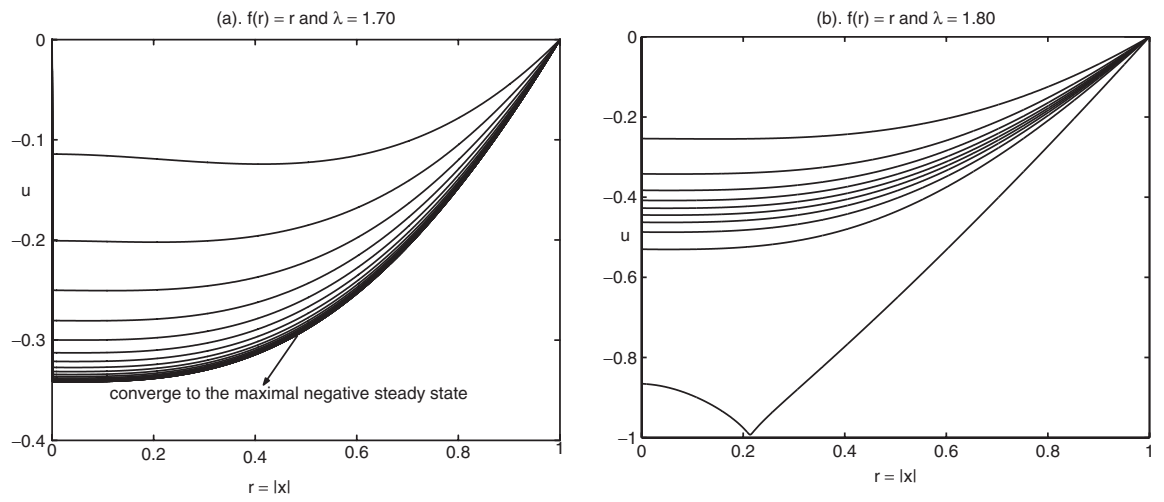

Figure 3 Left figure: for $\lambda=1.70$ we plot $u$ versus $r$ at different times showing the approach to the maximal negative steady-state. Right figure: for $\lambda=1.80$ we plot $u$ versus $x$ at different times $t=0,0.4475,0.8950,1.3426,1.7901,2.2376,2.6851,3.1326,3.5802,4.0277,4.4751942$, from which touchdown is observed at $r=0.21361$. For both cases, we consider (3.1) with $f(r)=r$ in the unit disk domain.

Without loss of generality, we may assume $0 \in K \subset \bar{\Omega}$. Let $B_{r}=B_{r}(0) \subset K$ be the ball centered at $x=0$ and radius $r$. For $0<r_{1}<r_{2} \leq 4 r_{1}$, let $\eta(x) \in$ $C_{0}^{\infty}\left(B_{r_{2}}\right)$ be such that $\eta \equiv 1$ in $B_{r_{1}}, 0 \leq \eta \leq 1$ in $B_{r_{2}} \backslash B_{r_{1}}$ and $|\nabla \eta| \leq 2 /\left(r_{2}-r_{1}\right)$. Multiplying (3.3) by $\phi^{2} /(1-u)$, where $\phi=\eta^{\alpha}$ and $\alpha \geq 1$ is to be determined later, and integrating by parts we have

$$
\int_{B_{r_{2}}} \frac{m \phi^{2}}{(1-u)^{3}} \leq \int_{B_{r_{2}}} \frac{\phi^{2} \Delta u}{1-u}=-\int_{B_{r_{2}}} \frac{\phi^{2}|\nabla u|^{2}}{(1-u)^{2}}-\int_{B_{r_{2}}} \frac{2 \phi \nabla \phi \cdot \nabla u}{1-u} .
$$

From the fact,

$$
\begin{aligned}
\int_{B_{r_{2}}} \frac{2 \phi \nabla \phi \cdot \nabla u}{1-u} & \leq \int_{B_{r_{2}}} \phi^{2}|\nabla u|^{2}+4 \int_{B_{r_{2}}} \frac{|\nabla \phi|^{2}}{(1-u)^{2}} \\
& \leq \int_{B_{r_{2}}} \frac{\phi^{2}|\nabla u|^{2}}{(1-u)^{2}}+4 \int_{B_{r_{2}}} \frac{|\nabla \phi|^{2}}{(1-u)^{2}},
\end{aligned}
$$

(3.4) gives that

$$
\int_{B_{r_{2}}} \frac{m \phi^{2}}{(1-u)^{3}} \leq 4 \int_{B_{r_{2}}} \frac{|\nabla \phi|^{2}}{(1-u)^{2}}
$$

Now choose $\phi=\eta^{2 \beta}$ with $\beta=\frac{3}{2}$. Then Hölder's inequality implies that

$$
m \int_{B_{r_{2}}} \frac{\eta^{4 \beta}}{(1-u)^{3}} \leq 16 \beta^{2}\left[\int_{B_{r_{2}}}|\nabla \eta|^{4 \beta}\right]^{\frac{1}{2 \beta}}\left[\int_{B_{r_{2}}} \frac{\eta^{4 \beta}}{(1-u)^{3}}\right]^{\frac{2 \beta-1}{2 \beta}}
$$


This shows that

$$
\int_{B_{r_{1}}} \frac{1}{(1-u)^{3}} \leq \int_{B_{r_{2}}} \frac{\eta^{4 \beta}}{(1-u)^{3}}<C\left(m, r_{1}\right) .
$$

By virtue of the one-sided Harnack inequality, we have

$$
\left\|\frac{1}{1-u}\right\|_{L^{\infty}\left(B \frac{r_{1}}{2}\right)}=\|v\|_{L^{\infty}\left(B \frac{r_{1}}{2}\right)} \leq C\left(r_{1}\right)\|v\|_{L^{3}\left(B_{r_{1}}\right)}<C\left(r_{1}, m\right) .
$$

The rest follows from a standard compactness argument.

Proof of Theorem 3.1: Set $v=u_{t}$, then we have for any $t_{1}<T$ that

$$
\begin{gathered}
v_{t}=\Delta v+\frac{2 \lambda f(x)}{(1-u)^{3}} v \quad(x, t) \in \Omega \times\left(0, t_{1}\right) ; \\
v(x, t)=0 \text { for }(x, t) \in \partial \Omega \times\left(0, t_{1}\right) \quad \text { and } \quad v(x, 0) \geq 0 \text { for } x \in \Omega .
\end{gathered}
$$

Note that the term $\frac{2 \lambda f}{(1-u)^{3}}$ is locally bounded in $\Omega \times\left(0, t_{1}\right)$, so that by the strong maximum principle, we may conclude

$$
u_{t}=v>0 \text { for }(x, t) \in \Omega \times\left(0, t_{1}\right)
$$

and therefore, $u_{t}>0$ holds for all $(x, t) \in \Omega \times(0, T)$. Since $K$ is an isolated set of touchdown points, there exists an open set $U$ such that $K \subset U \subset \bar{U} \subset \Omega$ with no

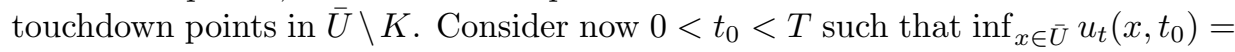
$C_{1}>0$. We claim that there exists $\varepsilon>0$ such that

$$
J^{\epsilon}(x, t)=u_{t}-\frac{\varepsilon}{(1-u)^{2}} \geq 0 \quad \text { for all } \quad(x, t) \in U \times\left(t_{0}, T\right),
$$

Indeed, there exists $C_{2}>0$ such that $u_{t}(x, T) \geq C_{2}>0$ on $U$, and since $\partial U$ has no touchdown points, there exists $\varepsilon>0$ such that $J^{\epsilon} \geq 0$ on the parabolic boundary of $U \times\left(t_{0}, T\right)$. Also, direct calculations imply that

$$
J_{t}^{\epsilon}-\Delta J^{\epsilon}=\frac{2 \lambda f}{(1-u)^{3}} J^{\epsilon}+\frac{6 \varepsilon|\nabla u|^{2}}{(1-u)^{4}} \geq \frac{2 \lambda f}{(1-u)^{3}} J^{\epsilon} .
$$

Since $\frac{\varepsilon}{(1-u)^{2}}$ is locally bounded on $U \times\left(t_{0}, T\right)$, we can apply the maximum principle to obtaining (3.8).

If now $\inf _{x \in K} f(x)=0$, then we may combine (3.8) and (1.1), to deduce that for a small neighborhood $B \subset U$ of some point $x_{0} \in K$ where $f(x) \leq \varepsilon / 2$, we have

$$
\Delta u \geq \frac{\varepsilon}{2} \frac{1}{(1-u)^{2}} \quad \text { for }(x, t) \in B \times\left(t_{0}, T\right) .
$$


In view of Lemma 3.2, this contradicts to the assumption that $x_{0}$ is a touchdown point.

For the second part, recall from Theorem B stated in the introduction that the unique extremal solution for the stationary problem on the ball in the case $N \geq 8$ and for a permittivity profile $f(x)=|x|^{\alpha}$, is $u^{*}(x)=1-|x|^{\frac{2+\alpha}{3}}$ as long as $\alpha$ is small enough. Theorem 2.5 then implies that the origin 0 is a touchdown point of the solution even though it is also a root for the permittivity profile (i.e., $f(0)=0)$. This complements the statement of Theorem 3.1 above. In other words, zero points of $f$ in $\Omega$ cannot be on the isolated set of touchdown points in finite time (which occur when $\lambda>\lambda^{*}$ ) but can very well be touchdown points in infinite time of (1.1), which can only happen when $\lambda=\lambda^{*}$. The proof of Theorem 3.1 fails for touchdowns in infinite time, simply because the maximum principle cannot be applied in the infinite cylinder $\Omega \times(0, \infty)$.

\section{Estimates for Finite Touchdown Times}

In this section we give comparaison results and explicit estimates on finite touchdown times of dynamic deflections $u=u(x, t)$ whenever $\lambda>\lambda^{*}$. This often translates into useful information concerning the speed of the operation for many MEMS devices such as RF switches or micro-valves.

\subsection{Comparison results for finite touchdown time}

We start by comparing the effect on the finite touchdown time of two different but comparable permittivity profiles $f(x)$, at a given voltage $\lambda$.

Theorem 4.1 Suppose $u_{1}=u_{1}(x, t)$ (resp., $u_{2}=u_{2}(x, t)$ ) is a touchdown solution for (1.1) associated to a fixed voltage $\lambda$ and permittivity profiles $f_{1}$ (resp., $f_{2}$ ) with a corresponding finite touchdown time $T_{\lambda}\left(\Omega, f_{1}\right)$ (resp., $T_{\lambda}\left(\Omega, f_{2}\right)$ ). If $f_{1}(x) \geq$ $f_{2}(x)$ on $\Omega$ and if $f_{1}(x)>f_{2}(x)$ on a set of positive measure, then necessarily $T_{\lambda}\left(\Omega, f_{1}\right)<T_{\lambda}\left(\Omega, f_{2}\right)$.

Proof. By making a change of variable $v=1-u$, we can assume to be working with solutions of the following equation:

$$
\begin{aligned}
\frac{\partial v}{\partial t} & =\Delta v-\frac{\lambda f(x)}{v^{2}} & & \text { for } & & x \in \Omega, \\
v(x, t) & =1 & & \text { for } & & x \in \partial \Omega, \\
v(x, 0) & =1 & & \text { for } & & x \in \Omega,
\end{aligned}
$$

where $f$ is either $f_{1}$ or $f_{2}$. Suppose now that $T_{\lambda}\left(\Omega, f_{1}\right)>T_{\lambda}\left(\Omega, f_{2}\right)$ and let $\Omega_{0} \subset \Omega$ be the set of touchdown points of $u_{2}$ at finite time $T_{\lambda}\left(\Omega, f_{2}\right)$. Setting $w=u_{2}-u_{1}$, 
we get that

$$
w_{t}-\Delta w-\frac{\lambda\left(f_{2} u_{1}+f_{1} u_{2}\right)}{u_{1}^{2} u_{2}^{2}} w=\frac{\lambda\left(f_{1}-f_{2}\right)}{u_{1} u_{2}} \geq 0 \quad(x, t) \in \Omega \times\left(0, T_{\lambda}\left(\Omega, f_{2}\right)\right) .
$$

Since $w=0$ at $t=0$ as well as on $\partial \Omega \times\left(0, T_{\lambda}\left(\Omega, f_{2}\right)\right)$, we get from the maximum principle that $w$ cannot attain a negative minimum in $\Omega \times\left(0, T_{\lambda}\left(\Omega, f_{2}\right)\right)$, and therefore $w \geq 0$ in $\Omega \times\left(0, T_{\lambda}\left(\Omega, f_{2}\right)\right)$. Since $u_{2} \rightarrow 0$ in $\Omega_{0}$ as $t \rightarrow T_{\lambda}\left(\Omega, f_{2}\right)$, and since our assumption is that $T_{\lambda}\left(\Omega, f_{1}\right)>T_{\lambda}\left(\Omega, f_{2}\right)$, we then have $u_{1}>0$ in $\Omega_{0}$ as $t \rightarrow T_{\lambda}\left(\Omega, f_{2}\right)$. Therefore, $w<0$ in $\Omega_{0}$ as $t \rightarrow T_{\lambda}\left(\Omega, f_{2}\right)$, which is a contradiction and therefore $T_{\lambda}\left(\Omega, f_{1}\right) \leq T_{\lambda}\left(\Omega, f_{2}\right)$.

To prove the strict inequality, we note that the above proof shows that $w \geq 0$ in $\Omega \times\left(0, T_{\lambda}\left(\Omega, f_{2}\right)\right)$, which once combined with (4.2) gives that

$$
w_{t}-\Delta w \geq 0, \quad \text { in } \Omega \times\left(t_{1}, T_{\lambda}\left(\Omega, f_{2}\right)\right),
$$

where $t_{1}>0$ is chosen so that $w\left(x, t_{1}\right) \not \equiv 0$ in $\Omega$. Now we compare $w$ with the solution $z$ of

$$
z_{t}-\Delta z=0 \quad \text { in } \quad \Omega \times\left(t_{1}, T_{\lambda}\left(\Omega, f_{2}\right)\right)
$$

subject to $z\left(x, t_{1}\right)=w\left(x, t_{1}\right)$ and $z(x, t)=0$ on $\partial \Omega \times\left(t_{1}, T_{\lambda}\left(\Omega, f_{2}\right)\right)$. Clearly, $w \geq z$ in $\Omega \times\left(t_{1}, T_{\lambda}\left(\Omega, f_{2}\right)\right)$. On the other hand, for any $t_{0}>t_{1}$ we have $z>$ 0 in $\Omega \times\left(t_{0}, T_{\lambda}\left(\Omega, f_{2}\right)\right)$. Consequently, $w>0$ which means that $u_{2}>u_{1}$ in $\Omega \times\left(t_{0}, T_{\lambda}\left(\Omega, f_{2}\right)\right)$ and therefore $T_{\lambda}\left(\Omega, f_{1}\right)<T_{\lambda}\left(\Omega, f_{2}\right)$.

The second comparison result deals with different applied voltages but identical permittivity profiles.

Theorem 4.2 Suppose $u_{1}=u_{1}(x, t)$ (resp., $u_{2}=u_{2}(x, t)$ ) is a solution for (1.1) associated to a voltage $\lambda_{1}$ (resp., $\lambda_{2}$ ) and which has a finite touchdown time $T_{\lambda_{1}}(\Omega, f)$ (resp., $T_{\lambda_{2}}(\Omega, f)$ ). If $\lambda_{1}>\lambda_{2}$, then necessarily $T_{\lambda_{1}}(\Omega, f)<T_{\lambda_{2}}(\Omega, f)$.

Proof. It is similar to the proof of Theorem 4.1, except that for $w=u_{2}-u_{1},(4.2)$ is replaced by

$$
w_{t}-\Delta w-\frac{\lambda_{1}\left(u_{1}+u_{2}\right) f}{u_{1}^{2} u_{2}^{2}} w=\frac{\left(\lambda_{1}-\lambda_{2}\right) f}{u_{2}^{2}} \geq 0 \quad(x, t) \in \Omega \times(0, T) .
$$

The details are left for the interested reader.

Remark 4.1 A reasoning similar to the one found in Proposition 2.5 of [9], gives some information on the dependence on the shape of the domain. Indeed, for any bounded domain $\Gamma$ in $\mathbb{R}^{N}$ and any non-negative continuous function $f$ on $\Gamma$, we have

$$
\lambda^{*}(\Gamma, f) \geq \lambda^{*}\left(B_{R}, f^{*}\right) \text { and } T_{\lambda}(\Gamma, f) \geq T_{\lambda}\left(B_{R}, f^{*}\right)
$$



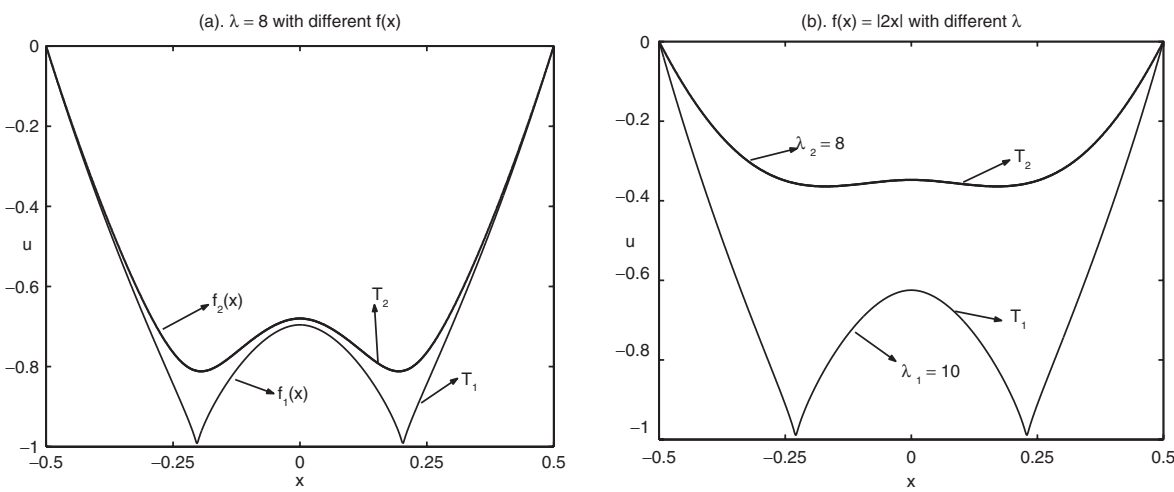

Figure 4 Left figure: plots of $u$ versus $x$ with $\lambda=8$ for different profiles $f(x)$ at the time $t=0.185736$ in the slab domain. The finite touchdown time $T_{\lambda}\left(\Omega, f_{1}\right)$ for the case $f_{1}(x)=|2 x|$ and $T_{\lambda}\left(\Omega, f_{2}\right)$ for the case $f_{2}(x)$ defined by $(4.3)$ are 0.185736 and 0.186688 , respectively. Right figure: plots of $u$ versus $x$ at the time $t=0.1254864$ for $f(x)=|2 x|$ with different values of $\lambda$ in the slab domain. The finite touchdown time $T_{\lambda_{1}}(\Omega, f)$ for the case $\lambda_{1}=10$ and $T_{\lambda_{2}}(\Omega, f)$ for the case $\lambda_{2}=8$ are 0.1254864 and 0.185736 , respectively.

where $B_{R}=B_{R}(0)$ is the Euclidean ball in $\mathbb{R}^{N}$ with radius $R>0$ and with volume $\left|B_{R}\right|=|\Gamma|$, where $f^{*}$ is the Schwarz symmetrization of $f$.

We now present numerical results comparing finite touchdown times in a slab domain.

Fig. 4(a): Dependence on the dielectric permittivity profiles $f$ We consider (3.1) for the cases where

$$
f_{1}(x)=|2 x| \quad \text { and } \quad f_{2}(x)=\left\{\begin{array}{cl}
|2 x| & \text { if }|x| \leq \frac{1}{8} \\
1 / 4+2 \sin (|x|-1 / 8) & \text { otherwise. }
\end{array}\right.
$$

Using $N=1000$ meshpoints, we plot $u$ versus $x$ with $\lambda=8$ at the time $t=$ 0.185736 in Fig. 4(a). The numerical results show that the finite touchdown time $T_{\lambda}\left(\Omega, f_{1}\right)$ for the case $f_{1}(x)$ and $T_{\lambda}\left(\Omega, f_{2}\right)$ for the case $f_{2}(x)$ are 0.185736 and 0.186688 , respectively.

Fig. 4(b): Dependence on the applied voltage $\lambda$

Using $N=1000$ meshpoints and the profile $f(x)=|2 x|$, we plot $u$ of (3.1) versus $x$ with different values of $\lambda$ at the time $t=0.1254864$. The numerical results show that finite touchdown time $T_{\lambda_{1}}(\Omega, f)$ for applied voltage $\lambda_{1}=10$ and $T_{\lambda_{2}}(\Omega, f)$ for applied voltage $\lambda_{2}=8$ are 0.1254864 and 0.185736 , respectively.

\subsection{Explicit bounds on finite touchdown times}

We now establish claims 1), 3) and 4) in Theorem 1.3 of the introduction. 
Proposition 4.3 Suppose $f$ is a function satisfying (1.2) on a bounded domain $\Omega$, then,

1. For $\lambda>0$, we have $T_{\lambda}(\Omega, f) \geq T_{*}:=\frac{1}{3 \lambda \sup _{x \in \Omega} f(x)}$.

2. If $\inf _{\Omega} f>0$, and if $\lambda>\bar{\lambda}_{1}:=\frac{4 \mu_{\Omega}}{27 \inf _{x \in \Omega} f(x)}$, then

$$
T_{\lambda}(\Omega, f) \leq T_{1, \lambda}(\Omega, f):=\int_{0}^{1}\left[\frac{\lambda \inf _{x \in \Omega} f(x)}{(1-s)^{2}}-\mu_{\Omega} s\right]^{-1} d s .
$$

3. If $f>0$ on a set of positive measure, and if $\lambda>\bar{\lambda}_{2}:=\frac{\mu_{\Omega}}{3 \int_{\Omega} f \phi_{\Omega} d x}$, then

$$
T_{\lambda}(\Omega, f) \leq T_{2, \lambda}(\Omega, f):=-\frac{1}{\mu_{\Omega}} \log \left[1-\frac{\mu_{\Omega}}{3 \lambda}\left(\int_{\Omega} f \phi_{\Omega} d x\right)^{-1}\right]
$$

Proof. 1) Consider the initial value problem:

$$
\begin{aligned}
\frac{d \eta(t)}{d t} & =\frac{\lambda M}{(1-\eta(t))^{2}}, \\
\eta(0) & =0
\end{aligned}
$$

where $M=\sup _{x \in \Omega} f(x)$. From (4.6) one has $\frac{1}{\lambda M} \int_{0}^{\eta(t)}(1-s)^{2} d s=t$. If $T_{*}$ is the time where $\lim _{t \rightarrow T_{*}} \eta(t)=1$, then we have $T_{*}=\frac{1}{\lambda M} \int_{0}^{1}(1-s)^{2} d s=\frac{1}{3 \lambda M}$. Obviously, $\eta(t)$ is now a super-function of $u(x, t)$ near touchdown, and thus we have $T \geq T_{*}=\frac{1}{3 \lambda M}=\frac{1}{3 \lambda \sup _{x \in \Omega} f(x)}$. This completes the proof of 1$)$.

The following analytic upper bounds of finite touchdown time $T$ were established in Theorem 3.1 and 3.2 of [11]. We sketch their easy proofs for completeness.

2) Multiplying (1.1a) by $\phi_{\Omega}$, the first normalized eigenfunction of $-\Delta$, and integrating over the domain, we obtain

$$
\frac{d}{d t} \int_{\Omega} \phi_{\Omega} u d x=\int_{\Omega} \phi_{\Omega} \Delta u d x+\int_{\Omega} \frac{\lambda \phi_{\Omega} f(x)}{(1-u)^{2}} d x .
$$

Using Green's theorem, together with the lower bound $C_{0}$ of $f$, we get

$$
\frac{d}{d t} \int_{\Omega} \phi_{\Omega} u d x \geq-\mu_{\Omega} \int_{\Omega} \phi_{\Omega} u d x+\lambda C_{0} \int_{\Omega} \frac{\phi_{\Omega}}{(1-u)^{2}} d x .
$$

Next, we define an energy-like variable $E(t)$ by $E(t)=\int_{\Omega} \phi_{\Omega} u d x$ so that

$$
E(t)=\int_{\Omega} \phi_{\Omega} u d x \leq \sup _{\Omega} u \int_{\Omega} \phi_{\Omega} d x=\sup _{\Omega} u \text {. }
$$


Moreover, $E(0)=0$ since $u=0$ at $t=0$. Then, using Jensen's inequality on the second term on the right-hand side of (4.8), we obtain

$$
\frac{d E}{d t}+\mu_{\Omega} E \geq \frac{\lambda C_{0}}{(1-E)^{2}}, \quad E(0)=0 .
$$

We then compare $E(t)$ with the solution $F(t)$ of

$$
\frac{d F}{d t}+\mu_{\Omega} F=\frac{\lambda C_{0}}{(1-F)^{2}}, \quad F(0)=0 .
$$

Standard comparison principles yield that $E(t) \geq F(t)$ on their domains of existence. Therefore,

$$
\sup _{\Omega} u \geq E(t) \geq F(t) .
$$

Next, we separate variables in (4.11) to determine $t$ in terms of $F$, and it is easy to see that the touchdown time $\bar{T}_{1}$ for $F$ is given by

$$
\bar{T}_{1} \equiv \int_{0}^{1}\left[\frac{\lambda C_{0}}{(1-s)^{2}}-\mu_{\Omega} s\right]^{-1} d s .
$$

Note that $\bar{T}_{1}$ is finite whenever the integral in (4.13) converges, and a simple calculation shows that this occurs whenever $\lambda>\bar{\lambda}_{1} \equiv \frac{4 \mu_{\Omega}}{27 C_{0}}$. Moreover, if $\bar{T}_{1}$ is finite, then (4.12) implies that the touchdown time $T$ of (1.1) must also be finite. It follows that when $\lambda>\bar{\lambda}_{1}=\frac{4 \mu_{\Omega}}{27 C_{0}}$, we have

$$
T_{\lambda}(\Omega, f) \leq \bar{T}_{1} \equiv \int_{0}^{1}\left[\frac{\lambda C_{0}}{(1-s)^{2}}-\mu_{\Omega} s\right]^{-1} d s .
$$

3) Multiply now (1.1a) by $\phi_{\Omega}(1-u)^{2}$, and integrate the resulting equation over $\Omega$ to get

$$
\frac{d}{d t} \int_{\Omega} \frac{\phi_{\Omega}}{3}(1-u)^{3} d x=-\int_{\Omega} \phi_{\Omega}(1-u)^{2} \Delta u d x-\int_{\Omega} \lambda f \phi_{\Omega} d x .
$$

We calculate the first term on the right-hand side of (4.15) to get

$$
\begin{aligned}
\frac{d}{d t} & \int_{\Omega} \frac{\phi_{\Omega}}{3}(1-u)^{3} d x \\
& =\int_{\Omega} \nabla u \cdot \nabla\left[\phi_{\Omega}(1-u)^{2}\right] d x+\int_{\partial \Omega}(1-u)^{2} \phi_{\Omega} \nabla u \cdot \hat{n} d S-\int_{\Omega} \lambda f \phi_{\Omega} d x \\
& =-\int_{\Omega} 2(1-u) \phi_{\Omega}|\nabla u|^{2} d x-\int_{\Omega} \frac{1}{3} \nabla \phi_{\Omega} \cdot \nabla\left[(1-u)^{3}\right] d x-\int_{\Omega} \lambda f \phi_{\Omega} d x \\
& \leq-\frac{1}{3} \int_{\partial \Omega} \nabla \phi_{\Omega} \cdot \hat{n} d S-\frac{\mu_{\Omega}}{3} \int_{\Omega}(1-u)^{3} \phi_{\Omega} d x-\int_{\Omega} \lambda f \phi_{\Omega} d x
\end{aligned}
$$


where $\hat{n}$ is the unit outward normal to $\partial \Omega$. Since $\int_{\partial \Omega} \nabla \phi_{\Omega} \cdot \hat{n} d S=-\mu_{\Omega}$, we further estimate from (4.16c) that

$$
\frac{d E}{d t}+\mu_{\Omega} E \leq R, \quad R \equiv \frac{\mu_{\Omega}}{3}-\lambda \int_{\Omega} f \phi_{\Omega} d x,
$$

where $E(t)$ is defined by

$$
E(t) \equiv \frac{1}{3} \int_{\Omega} \phi_{\Omega}(1-u)^{3} d x, \quad \text { with } \quad E(0)=\frac{1}{3} .
$$

Next, we compare $E(t)$ with the solution $F(t)$ of

$$
\frac{d F}{d t}+\mu_{\Omega} F=R, \quad F(0)=\frac{1}{3} .
$$

Again, comparison principles and the definition of $E$ yield

$$
\frac{1}{3} \inf _{\Omega}(1-u)^{3} \leq E(t) \leq F(t) .
$$

For $\lambda>\bar{\lambda}_{2}$ we have that $R<0$ in (4.17) and (4.19), and for $R<0$, we have necessarily that $F=0$ at some finite time $\bar{T}_{2}$ which, in view of (4.20), implies that $E=0$ at finite time. Thus, $u$ must touchdown at some finite time $T<\bar{T}_{2}$. By estimating $\bar{T}_{2}$ explicitly we get that

$$
T_{\lambda}(\Omega, f) \leq \bar{T}_{2} \equiv-\frac{1}{\mu_{\Omega}} \log \left[1-\frac{\mu_{\Omega}}{3 \lambda}\left(\int_{\Omega} f \phi_{\Omega} d x\right)^{-1}\right] .
$$

Remark 4.2 It follows from the above that if $\lambda>\max \left\{\bar{\lambda}_{1}, \bar{\lambda}_{2}\right\}$, and $\inf _{x \in \Omega} f(z)$ $>0$ then

$$
T_{\lambda}(\Omega, f) \leq \min \left\{T_{0, \lambda}, T_{1, \lambda}, T_{2, \lambda}\right\},
$$

where $T_{0, \lambda}$ is given by Theorem 2.3. We note that the three estimates on the touchdown times are not comparable. Indeed, it is clear that $T_{0, \lambda}$ is the better estimate when $\lambda^{*}<\lambda<\min \left\{\bar{\lambda}_{1}, \bar{\lambda}_{2}\right\}$, since $T_{1, \lambda}$ and $T_{2, \lambda}$ are not finite. On the other hand, our numerical simulations show that $T_{0, \lambda}$ can be much worse than the others for $\lambda>\max \left\{\bar{\lambda}_{1}, \bar{\lambda}_{2}\right\}$.

Here are now some numerical estimates for touchdown times for several choices of the domain $\Omega$ given by (3.2) and the exponential profile $f(x)$ satisfying

$$
\begin{array}{rll}
\text { (Slab) : } & f(x)=e^{\alpha\left(x^{2}-1 / 4\right)} & \text { (exponential), } \\
\text { (Unit Disk) : } & f(x)=e^{\alpha\left(|x|^{2}-1\right)} & \text { (exponential), }
\end{array}
$$




\begin{tabular}{c|c|c|c|c|c}
\hline$\Omega$ & $\alpha$ & $\underline{\lambda}$ & $\lambda^{*}$ & $\lambda_{1}$ & $\lambda_{2}$ \\
\hline (Slab) & 0 & 1.185 & 1.401 & 1.462 & 3.290 \\
(Slab) & 1.0 & 1.185 & 1.733 & 1.878 & 4.023 \\
(Slab) & 3.0 & 1.185 & 2.637 & 3.095 & 5.965 \\
(Slab) & 6.0 & 1.185 & 4.848 & 6.553 & 10.50 \\
(Unit Disk) & 0 & 0.593 & 0.789 & 0.857 & 1.928 \\
(Unit Disk) & 0.5 & 0.593 & 1.153 & 1.413 & 2.706 \\
(Unit Disk) & 1.0 & 0.593 & 1.661 & 2.329 & 3.746 \\
(Unit Disk) & 3.0 & 0.593 & 6.091 & 17.21 & 11.86 \\
\hline
\end{tabular}

Table 1 Numerical values for pull-in voltage $\lambda^{*}$ with the bounds $\underline{\lambda}, \bar{\lambda}_{1}$ and $\bar{\lambda}_{2}$ given in Theorem A. Here the exponential permittivity profile is chosen as (4.23).

where $\alpha \geq 0$. In order to choose proper applied voltage $\lambda$ satisfying $\lambda>\lambda^{*}$, we first compute the bounds $\bar{\lambda}_{1}$ and $\bar{\lambda}_{2}$. This requires to calculate the smallest eigenpair $\mu_{\Omega}$ and $\phi_{\Omega}$ of $-\Delta$, normalized by $\int_{\Omega} \phi_{\Omega} d x=1$ for either of the domains. A simple calculation yields that

$$
\begin{array}{ll}
\mu_{\Omega}=\pi^{2}, \quad & \phi_{\Omega}=\frac{\pi}{2} \sin \left[\pi\left(x+\frac{1}{2}\right)\right], \quad \text { (Slab) }, \\
\mu_{\Omega}=z_{0}^{2} \approx 5.783, \quad \phi_{\Omega}=\frac{z_{0}}{J_{1}\left(z_{0}\right)} J_{0}\left(z_{0}|x|\right), \quad(\text { Unit Disk }) .
\end{array}
$$

Here $J_{0}$ and $J_{1}$ are Bessel functions of the first kind, and $z_{0} \approx 2.4048$ is the first zero of $J_{0}(z)$. The bounds $\bar{\lambda}_{1}$ and $\bar{\lambda}_{2}$ can be evaluated by substituting (4.24) into (1.3). Notice that $\bar{\lambda}_{2}$ is, in general, determined only up to a numerical quadrature.

\begin{tabular}{c|c|c|c|c|c|c}
\hline$\Omega$ & $\alpha$ & $T_{*}$ & $T$ & $T_{0, \lambda}$ & $T_{1, \lambda}$ & $T_{2, \lambda}$ \\
\hline (Slab) & 0 & $1 / 60$ & 0.01668 & 0.2555 & 0.0175 & 0.01825 \\
(Slab) & 1.0 & $1 / 60$ & 0.02096 & $\leq 0.3383$ & 0.0229 & 0.02275 \\
(Slab) & 3.0 & $1 / 60$ & 0.03239 & $\leq 0.6121$ & 0.0395 & 0.03588 \\
(Slab) & 6.0 & $1 / 60$ & 0.06312 & $\leq 1.7033$ & 0.0973 & 0.07544 \\
(Unit Disk) & 0 & $1 / 60$ & 0.01667 & 0.2420 & 0.0172 & 0.01745 \\
(Unit Disk) & 0.5 & $1 / 60$ & 0.02241 & $\leq 0.4103$ & 0.0289 & 0.02507 \\
(Unit Disk) & 1.0 & $1 / 60$ & 0.02927 & $\leq 0.7123$ & 0.0492 & 0.03579 \\
(Unit Disk) & 3.0 & $1 / 60$ & 0.09563 & $\leq 8.9847$ & 1.1614 & 0.15544 \\
\hline
\end{tabular}

Table 2 Numerical values for finite touchdown time $T$ with the bounds $T_{*}, T_{0, \lambda}, T_{1, \lambda}$ and $T_{2, \lambda}$ given in Proposition 3.3. Here applied voltage $\lambda=20$ and the exponential permittivity profile is chosen as (4.23).

In Table 1 we give numerical results for the saddle-node value $\lambda^{*}$ with the bounds $\underline{\lambda}, \bar{\lambda}_{1}$ and $\bar{\lambda}_{2}$ given in Theorem A, for the exponential permittivity profile 


\begin{tabular}{c|c|c|c|c}
\hline$\Omega$ & $T(\lambda=5)$ & $T(\lambda=10)$ & $T(\lambda=15)$ & $(\lambda=20)$ \\
\hline (Slab) & 0.07495 & 0.03403 & 0.02239 & 0.01668 \\
(Unit Disk) & 0.06699 & 0.03342 & 0.02235 & 0.01667 \\
\hline
\end{tabular}

Table 3 Numerical values for finite touchdown time $T$ at different applied voltages $\lambda=5,10$ 15 and 20, respectively. Here the constant permittivity profile $f(x) \equiv 1$ is chosen.

chosen as (4.23). These numerical results and Fig. 5 in [11] show that the pull-in voltage $\lambda^{*}$ is seen to increase with $\alpha$. Therefore, by increasing $\alpha$, or equivalently by increasing the spatial extent where $f(x) \ll 1$, one can increase the stable operating range of the MEMS capacitor. From Table 1 we also observe that the bound $\bar{\lambda}_{1}$ for $\lambda^{*}$ is better than $\bar{\lambda}_{2}$ just for small values of $\alpha$. For $\alpha \gg 1$, we use Laplace's method on the integral defining $\bar{\lambda}_{2}$, to obtain for this exponential permittivity profile that

$$
\bar{\lambda}_{1} \sim \frac{4 b_{1}}{27} e^{c_{1} \alpha}, \quad \bar{\lambda}_{2} \sim c_{2} \alpha^{2}
$$

Here $b_{1}=\pi^{2}, c_{1}=1 / 4, c_{2}=1 / 3$ for the slab domain, and $b_{1}=z_{0}^{2}, c_{1}=1$, $c_{2}=4 / 3$ for the unit disk, where $z_{0}$ is the first zero of $J_{0}(z)$. Therefore, for $\alpha \gg 1$ the bound $\bar{\lambda}_{2}$ is better than $\bar{\lambda}_{1}$.

Following the numerical results of Table 1, we can compute in Table 2 the values of finite touchdown time $T$ at $\lambda=20$, with the bounds $T_{*}, T_{0, \lambda}, T_{1, \lambda}$ and $T_{2, \lambda}$ given in Theorem 2.2 and Proposition 3.3. Using the meshpoints $N=800$ we compute finite touchdown time $T$ with error less than 0.00001 . The numerical results in Table 2 show that the bounds $T_{1, \lambda}$ and $T_{2, \lambda}$ for $T$ are much better than $T_{0, \lambda}$. Further the bound $T_{1, \lambda}$ is better than $T_{2, \lambda}$ for smaller values of $\alpha$, and however the bound $T_{2, \lambda}$ is better than $T_{1, \lambda}$ for larger values of $\alpha$. In fact, for $\alpha \gg 1$ and $\lambda$ large enough we can deduce from (4.25) that

$$
T_{1, \lambda} \sim \frac{1}{3 \lambda} e^{d_{1} \alpha}, \quad T_{2, \lambda} \sim \frac{d_{2}}{\lambda} \alpha^{2} .
$$

Here $d_{1}=1 / 4, d_{2}=1 / 3 \pi^{2}$ for the slab domain, and $d_{1}=1, d_{2}=4 / 3 z_{0}^{2}$ for the unit disk, where $z_{0}$ is the first zero of $J_{0}(z)=0$. Therefore, for $\alpha \gg 1$ and fixed $\lambda$ large enough, the bound $T_{2, \lambda}$ is better than $T_{1, \lambda}$. Table 2 also shows that for fixed applied voltage $\lambda$, the touchdown time is seen to increase once $\alpha$ is increased or equivalently the spatial extent where $f(x) \ll 1$ is increased. However, Theorem 3.2 tells us that for fixed permittivity profile $f$, by increasing the applied voltage $\lambda$ within the available power supply, the touchdown time can be decreased and consequently the operating speed of MEMS devices can be improved. In Table 3 we give numerical values for finite touchdown time $T$ with error less than 0.00001 , at different applied voltages $\lambda=5,10,15$ and 20, respectively. Here the constant permittivity profile $f(x) \equiv 1$ is chosen and the meshpoints $N=800$ again. 


\section{References}

[1] U. ASCHER, R. CHRISTIANSEN and R. RUSSELL, Collocation software for boundary value ODE's, Math. Comp. 33 (1979), 659-679.

[2] C. BANDLE, Isoperimetric inequalities and applications. In Monographs and Studies in Mathematics, Boston, Mass.-London, Pitman, 1980.

[3] R. E. BANK, PLTMG: A software package for solving elliptic partial differential equations, User's guide 8.0, Software, Environments, and Tools, SIAM, Philadelphia, PA, 1998, xi+110 pages.

[4] H. BELLOUT, A criterion for blow-up of solutions to semilinear heat equations, SIAM, J. Math. Anal. 18 (1987), 722-727.

[5] H. BREZIS, T. CAZENAVE, Y. MARTEL and A. RAMIANDRISOA, Blow up for $u_{t}-\Delta u=g(u)$ revisited, Adv. Diff. Eqns. (1996), 73-90.

[6] P. ESPOSITO, N. GHOUSSOUB and Y. GUO, Compactness along the first branch of unstable solutions for an elliptic problem with a singular nonlinearity, Comm. Pure Appl. Math. (2006) (In press).

[7] G. FLORES, G. A. MERCADO and J. A. PELESKO, Dynamics and touchdown in electrostatic MEMS, Proceedings of ICMENS 2003 (2003), 182-187.

[8] B. GIDAS, W. M. NI and L. NIRENBERG, Symmetry and related properties via the maximum principle, Comm. Math. Phys. 68 (1979), 209-243.

[9] N. GHOUSSOUB and Y. GUO, On the partial differential equations of electrostatic MEMS devices: stationary case, SIAM, J. Math. Anal. 38 (2007), $1423-1449$.

[10] Y. GUO, On the partial differential equations of electrostatic MEMS devices III: refined touchdown behavior, submitted (2006).

[11] Y. GUO, Z. PAN and M. WARD, Touchdown and pull-in voltage behavior of a MEMS device with varying dielectric properties, SIAM, J. Appl. Math. 66 (2005), 309-338.

[12] D. GILBARG and N. S. TRUDINGER, Elliptic partial differential equations of second order, 2nd, Springer, Berlin, 1983.

[13] D. D. JOSEPH and T. S. LUNDGREN, Quasilinear Dirichlet problems driven by positive sources, Arch. Ration. Mech. Anal. 49 (1973), 241-268.

[14] O. A. LADYZENSKAJA, V. A. SOLONNIKOV and N. N. URALCEVA, Linear and Quasilinear Equations of Parabolic Type. Transl. Math. Monographs, Amer. Math. Soc. 23, 1968. 
[15] J. A. PELESKO, Mathematical modeling of electrostatic MEMS with tailored dielectric properties, SIAM J. Appl. Math. 62 (2002), 888-908.

[16] J. A. PELESKO and D. H. BERNSTEIN, Modeling MEMS and NEMS, Chapman Hall and CRC Press, 2002.

Received 11 September 2006; accepted 11 January 2007

To access this journal online: http://www.birkhauser.ch 